\title{
Nodeless wave functions, spiky potentials, and the description of a quantum system in a quantum environment
}

\author{
Jakub Kocák (1) and Axel Schild (1) \\ Laboratorium für Physikalische Chemie, ETH Zürich, 8093 Zürich, Switzerland
}

(Received 2 June 2021; accepted 22 July 2021; published 27 August 2021)

\begin{abstract}
A quantum system $\mathscr{Q}$ is characterized by a single potential $v$ and its eigenstates. While $v$ is usually postulated for a given physical problem, it represents the interaction with an implicit environment $\mathscr{E}$. We use the exact factorization to show how $v$ emerges if the quantum environment is explicitly taken into account. In general, each eigenstate of the supersystem $\mathscr{S}=\mathscr{Q} \cup \mathscr{E}$ corresponds to a different potential $v_{j}$ and state $\chi_{j}$ of $\mathscr{Q}$. Such a state $\chi_{j}$ typically has no nodes and is the ground state of $v_{j}$, even if the corresponding state of the supersystem is an excited state. There are however two exceptions. First, if the energy scale for exciting $\mathscr{Q}$ is much smaller than for exciting $\mathscr{E}$, the potentials $v_{j}$ are similar in shape and differ only by sharp spikes. An excitation of $\mathscr{S}$ can then be viewed as an excitation of $\mathscr{Q}$ with its environment being unaffected, and $\mathscr{Q}$ is approximately described by a single spikeless potential $v$ and its eigenstates. Second, $\chi_{j}$ can sometimes have exact nodes, e.g., due to the symmetry of the problem, and is the excited state of a spikeless potential. We explain and investigate the two cases with model systems to illustrate the intricacies of the separation of a quantum system from its environment. As an application, we use the equivalence of $\chi_{j}$ being either an excited state of a spikeless potential or the ground state of a spiky potential: For one-dimensional systems, we provide a method to calculate the location of the nodes of an excited state from the calculation of a ground-state wave function. This approach can also be conceptually useful for the computationally hard problem of calculating highly excited states or many-fermion systems.
\end{abstract}

DOI: 10.1103/PhysRevResearch.3.033194

\section{INTRODUCTION}

In standard nonrelativistic quantum mechanics, a quantum system $\mathscr{Q}$ is described by a Hamiltonian, its eigenstates, and eigenenergies. To set up the Hamiltonian, the energetic contributions to the system are defined as a sum of the kinetic energy of the particles in the system and potentials that the particles experience. Those potentials originate from an interaction with some environment $\mathscr{E}$ that is not part of $\mathscr{Q}$ itself. For example, the Hamiltonian for a particle $(\mathscr{Q})$ in a laser field contains a (scalar and/or vector) potential representing the interaction with photons $(\mathscr{E})$, and the potential describing the nuclear vibration $(\mathscr{Q})$ of a diatomic originates from the presence of the electrons $(\mathscr{E})$ in the molecule.

The description of the quantum system $\mathscr{Q}$ interacting via potentials with some implicit environment $\mathscr{E}$ has to emerge from a quantum description of the supersystem $\mathscr{S}$ composed of $\mathscr{Q}$ and $\mathscr{E}$. The sketch in Fig. 1 shows the arrangement schematically: The quantum environment $\mathscr{E}$ is described by a wave function $\phi(q ; Q)$ that depends on the coordinates $q$ of the particles in $\mathscr{E}$ and, parametrically as defined below, also on the coordinates $Q$ of the particles in $\mathscr{Q}$. This wave function leads to a scalar potential $v$ and a vector potential $A$ that act on

Published by the American Physical Society under the terms of the Creative Commons Attribution 4.0 International license. Further distribution of this work must maintain attribution to the author(s) and the published article's title, journal citation, and DOI. the particles in $\mathscr{Q}$. The quantum system $\mathscr{Q}$ in its environment $\mathscr{E}$ is then described by the wave function $\chi$.

A well-known example of this emergence is the treatment of a molecule within the Born-Oppenheimer approximation (BOA) [1]. In the BOA, the energy of a molecule composed of electrons and nuclei is obtained by solving a Schrödinger equation for the nuclei alone, and the electrons provide the potential for the nuclear vibrational states. The quantum system $\mathscr{Q}$ is the nuclei, the quantum environment $\mathscr{E}$ is the electrons, and different states of the molecule (the supersystem $\mathscr{S}$ ) belong approximately to excitations of $\mathscr{Q}$ alone; the potential for the nuclei and hence $\mathscr{E}$ is the same for multiple vibrational states. This is indicated in Fig. 1(b), where several states $\psi_{j}$ of $\mathscr{S}$ (the molecule) belong to the same state $\phi_{0}$ of $\mathscr{E}$ (the electrons) and hence lead to the same potentials $\left\{v_{0}, A_{0}\right\}$ for $\mathscr{Q}$ (the nuclei); the wave functions $\chi_{j}$ of $\mathscr{Q}$ are different eigenstates of this one set of potentials. The BOA works well for many molecules because of the mass difference between nuclei and electrons, which leads to an approximate separation and to different energy scales for the excitation of these two particle types.

Notwithstanding this, the BOA is an approximation. If we want to know how the description of $\mathscr{Q}$ in terms of a single Hamiltonian emerges from the description of $\mathscr{S}$, we need to turn to an exact method that yields the BOA as a limit. A suitable method is the separation of a wave function into a marginal and a conditional part, which was first studied some time ago [2-4] and which has recently been extended as exact factorization (EF) [5-7]. While the EF is extensively used for the molecular problem, e.g., for obtaining 

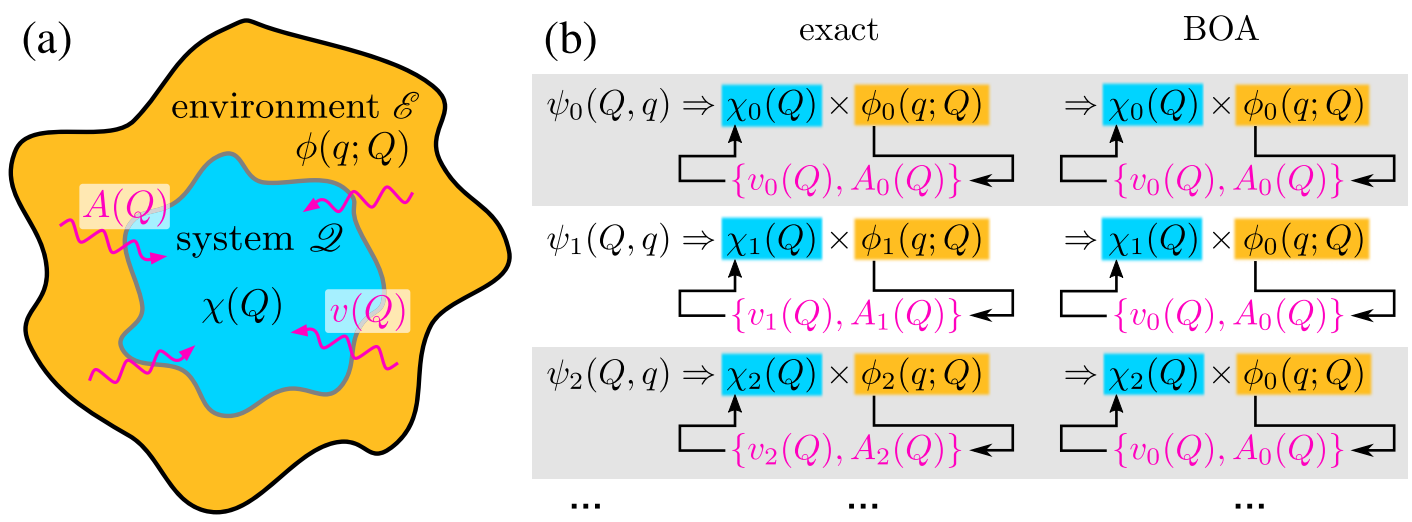

FIG. 1. (a) Quantum supersystem $\mathscr{S}$ consisting of the quantum system $\mathscr{Q}$ and the quantum environment $\mathscr{E}$. The wave function $\phi$ of $\mathscr{E}$ depends conditionally on $\mathscr{Q}$. The $\phi$ provides a scalar potential $v$ and a vector potential $A$ that encode the environmental effects of (the particles in) $\mathscr{E}$ on (the particles in) $\mathscr{Q}$. (b) Each state $\psi_{j}$ of $\mathscr{S}$ corresponds to one set of wave functions $\left\{\chi_{j}, \phi_{j}\right\}$ and their corresponding potentials $\left\{v_{j}, A_{j}\right\}$, as indicated in the column "exact." The $\chi_{j}$ are usually the ground states of these potentials. In the Born-Oppenheimer approximation (BOA), $\phi_{0}$ and hence also $\left\{v_{0}, A_{0}\right\}$ are approximately the same for multiple states, and the wave functions $\chi_{j}$ are excited states of these potentials.

the electron dynamics [8,9], as a trajectory-based simulation method [10-12], in the context of Bohmian mechanics [13], to find the effective nuclear mass due to the presence of the electrons [14], or to extend density functional theory $[15,16]$, many further applications have been found, such as the inverse electron-nuclear problem [17], treating light-matter interactions $[18,19]$, obtaining an embedding method for electronic wave functions [20] as well as reducing a many-electron problem to a one-electron problem [21-23], explaining the status of the time parameter in quantum mechanics [24-27], and more [28]. The many applications illustrate that the EF formalism is applicable to a wide variety of problems. Thus, we consider the EF here as a general method to separate a quantum system and an environment. As such, the EF is an alternative to other methods like density matrix theory $[29,30]$ and understanding how it describes the interaction of a system with its environment is useful to assess its applicability.

In the EF, the probability density $\left|\psi_{j}(Q, q)\right|^{2}$ for the wave function $\psi_{j}(Q, q)$ of the $j$ th state of the supersystem $\mathscr{S}$ is written as the product

$$
\left|\psi_{j}(Q, q)\right|^{2}=\left|\chi_{j}(Q)\right|^{2}\left|\phi_{j}(q ; Q)\right|^{2},
$$

where

$$
|\chi(Q)|^{2}=\int|\psi(Q, q)|^{2} d q
$$

is the marginal probability density of the system $\mathscr{Q}$ of particles with coordinates $Q$ and $\left|\phi_{j}(q ; Q)\right|^{2}$ is the conditional probability density of the environment $\mathscr{E}$ of particles with coordinates $q$, given some configuration $Q$. The relation (1) looks very similar to the BOA ansatz (where $Q$ are the nuclear coordinates and $q$ are the electronic coordinates), but no approximation or restriction of the type of particles is made. An important result of the EF, discussed below, is that if $\psi_{j}(Q, q)$ is given by a Schrödinger equation, also $\chi_{j}(Q)$ is given by a Schrödinger equation. In this Schrödinger equation, the environment $\mathscr{E}$ appears only as a scalar potential $v_{j}(Q)$ and a vector potential $A_{j}(Q)$, and both potentials are functionals of $\phi_{j}$. However, contrary to the usual description of a quantum system in terms of one potential and its eigenstates, every state $\psi_{j}$ corresponds to one wave function $\phi_{j}$ of the environment and hence also to one (not necessarily unique) set of potentials $\left\{v_{j}, A_{j}\right\}$. In the column "exact" in Fig. 1, this relationship between the wave functions and potentials is indicated schematically. Additionally, the state $\chi_{j}$ is only one eigenstate of $\left\{v_{j}, A_{j}\right\}$. Thus, in general it is not possible to describe $\mathscr{Q}$ with one set of potentials $\left\{v_{0}, A_{0}\right\}$ and several of its eigenstates, like in the BOA, but several potentials $\left\{v_{j}, A_{j}\right\}$ have to be used with one eigenstate for each of them. Finally, $\chi_{j}$ is usually the ground state of its potentials, even if the corresponding state $\psi_{j}$ is an excited state of the supersystem. The reason why this is the case is because $\chi_{j}$ typically has no nodes, as the condition for nodes to appear is rather strong: From (2) it follows that, at the position $Q_{0}$ of a possible node, $\psi\left(Q_{0}, q\right)$ needs to be zero for all values of $q$.

The first goal of this article is to illustrate how the EF separates the system and environment. We use two simple model systems to understand the peculiarities of the transition from the multipotential description of $\mathscr{Q}$ to the description in terms of one potential and its eigenstates.

In particular, when the exact states $\chi_{j}$ of $\mathscr{Q}$ are ground states of their potentials even if they belong to excited states of $\mathscr{S}$, they do not have nodes. Instead, in situations where the BOA becomes applicable, the scalar potentials $v_{j}$ of several states become similar to each other, with the exception of certain positions where the $v_{j}$ have spikes (on the scale of the problem; the $v_{j}$ are smooth functions, as explained below). Then the different $v_{j}$ can be replaced by one spikeless potential $v_{0}$ and the states $\chi_{j}$ can be obtained as excited states of this potential. In this way, the BOA recovers the standard treatment of a (closed) quantum system.

The appearance of nodeless wave functions and spiky potentials [4] has been discussed repeatedly [3,4,31-37]. In the literature, there is also some discussion about the existence of states $\chi_{j}$ with exact nodes, in particular for situations where a conical intersection appears in a Born-Oppenheimer treatment of the molecular problem [35-37]. The second goal of our article is to contribute to this discussion by presenting models constructed such that $\chi_{j}$ has nodes. We show that these wave functions can be interpreted as both ground and excited states 
of potentials with and without spikes, respectively. It follows that there is an equivalence of excited states of some potential $v_{0}$ with ground states of a potential $v_{j}$ that is almost $v_{0}$, except at isolated points, where spikes are found.

Our third goal with this article is to use this equivalence. We observe that, for one-dimensional problems, the nodes of the $j$ th eigenstate of $v_{0}$ are at the positions where the $(j-i)$ th eigenstate of $v_{i}(i \leqslant j)$ has its maximum energy as a function of the position of the spikes. An interesting consequence is an algorithm to compute the location of nodes of excited states from ground-state calculations. Although we only found a proof for one-dimensional problems, the idea may be useful in the context of the sign problem in quantum Monte Carlo methods that is still an obstacle for determining excited states or states of a fermionic problem [38].

To achieve the three goals, we start by describing the EF in Sec. II. Thereafter, in Sec. III we show how nodes develop for the wave functions of a quantum system $\mathscr{Q}$ in its environment, in the limit of the BOA, and why a gauge freedom in the theory of the EF is relevant. In Sec. IV we explain how these nodes correspond to spikes in the potentials and we present how the BOA picture of excited states of one spikeless potential emerges. The BOA is an approximation and the wave function of $\mathscr{Q}$ typically does not have exact nodes. In Sec. V we first discuss literature cases and we present two models where exact nodes can be found. There it becomes clear that excited states of a spikeless potential can be viewed as the ground state (or lower excited states) of a spiky potential. This is further investigated in Sec. VI and used to propose an algorithm for determining the location of nodes of excited states in one-dimensional problems. A conclusion is given in Sec. VII.

To avoid excessive definitions due to the different models used in the article, we use the notation of Sec. II, where the relevant quantities of the exact factorization are introduced, throughout the text for different models. The notation within a section is unique, but the same symbols are used for similar quantities in the different sections. Vector quantities do not have a special font except for the parameter vectors introduced in Sec. VI. The numerical solutions of the model systems are obtained with the PYTHON toolbox QMSTUNFTI [39] that uses the sparse matrix functionality of SCIPY [40], which in turn uses the ARPACK library [41] for solving eigenvalue problems. We use atomic units.

\section{EXACT FACTORIZATION}

The EF [5-7] is a method to separate a quantum system $\mathscr{Q}$ from its environment $\mathscr{E}$. Given that the supersystem $\mathscr{S}$ composed of $\mathscr{Q}$ and $\mathscr{E}$ is described by a Schrödinger equation, also $\mathscr{Q}$ is described by a Schrödinger equation. The EF explicitly provides the potentials that appear in the Hamiltonian of $\mathscr{Q}$ due to the presence of $\mathscr{E}$, and these potentials are given in terms of the wave function of $\mathscr{E}$. In the following, we use a two-particle notation and a simple kinetic energy operator for $\mathscr{S}$, but the formalism extends to any number of particles and to more complicated Hamiltonians.

Let $\psi(Q, q)$ be the wave function of a multicomponent quantum system $\mathscr{S}$ with coordinates $Q$ and $q$ that describe any number of (possibly identical) particles. The particles with coordinates $Q$ constitute the quantum (sub)system $\mathscr{Q}$ whose properties are to be determined in the presence of the particles at $q$, which represent the environment $\mathscr{E}$ of $\mathscr{Q}$. The wave function $\psi(Q, q)$ is written as a product

$$
\psi(Q, q)=\chi(Q) \phi(q ; Q),
$$

with the partial normalization condition

$$
\langle\phi(q ; Q) \mid \phi(q ; Q)\rangle_{q} \stackrel{!}{=} 1 \forall Q .
$$

Here $\langle a \mid b\rangle_{q}$ is the complex-valued scalar product with respect to the $q$ space. With these definitions and with the assumption that $\psi(Q, q)$ is normalized, (3) just states that a joint probability density is the product of a conditional probability density and a marginal probability density [see (1)] but in terms of probability amplitudes (wave functions). We write the wave function for $\mathscr{Q}$ explicitly as

$$
\chi(Q):=e^{-i S(Q)} \sqrt{\rho(Q)}, \quad S \in \mathbb{R},
$$

with the marginal density $\rho(Q) \equiv|\chi(Q)|^{2}$ given by (2). Here $\rho$ corresponds to the probability density for the quantum system $\mathscr{Q}$ in the presence of the particles in the environment $\mathscr{E}$. There is no ambiguity in the definition of $\rho(Q)$, but the wave function $\chi$ is uniquely defined only up to the phase $S(Q)$. This phase is a gauge freedom and therefore observables have to be defined such that they are independent of the choice of $S$, as discussed below.

The wave function for the environment $\mathscr{E}$ is the conditional wave function

$$
\phi(q ; Q):=\frac{\psi(Q, q)}{\chi(Q)},
$$

which, after choosing $S(Q)$ in (5), is also uniquely defined. It is a wave function that depends parametrically on $Q$, much like a time-dependent wave function depends on a parameter $t$ called time [24]. The $t$ parameter in time-dependent quantum mechanics is however a classical parameter, while the $Q$ parameter is a quantum parameter that is distributed according to $\chi(Q)$ [27].

The wave function $\psi(Q, q)$ is a solution of the Schrödinger equation

$$
\left(-\frac{\nabla_{Q}^{2}}{2 M}+H(Q)\right) \psi(Q, q)=E \psi(Q, q),
$$

with the Hamiltonian of $\mathscr{E}$ given as

$$
H(Q)=-\frac{\nabla_{q}^{2}}{2 m}+V(Q, q) .
$$

The notation $H(Q)$ emphasizes that $H$ is also a function of the coordinates $Q$ of particles that are not part of $\mathscr{E}$. With these definitions, it follows that the marginal wave function is also a solution of a Schrödinger equation, but for $\mathscr{Q}$ alone,

$$
\left(\frac{\left[-i \nabla_{Q}+A(Q)\right]^{2}}{2 M}+v(Q)\right) \chi(Q)=E \chi(Q),
$$

with a scalar potential

$$
v(Q)=v^{\mathrm{H}}(Q)+v^{\mathrm{G}}(Q)
$$


and a vector potential

$$
A(Q)=\left\langle\phi(q ; Q) \mid-i \nabla_{Q} \phi(q ; Q)\right\rangle_{q} .
$$

The scalar and vector potentials fully describe the effect that the environment $\mathscr{E}$ has on $\mathscr{Q}$ and are obtained from the wave function $\phi(q ; Q)$ of the environment. The scalar potential is composed of two terms. The first term is the average energy of the environment for a certain configuration $Q$ of the quantum system $\mathscr{Q}$,

$$
v^{\mathrm{H}}(Q)=\langle\phi(q ; Q)|H(Q)| \phi(q ; Q)\rangle_{q} .
$$

The second term, which originates from the kinetic energy operator with respect to $Q$, is the geometric term that originates from how the wave function $\phi(q ; Q)$ of the environment changes when the configuration $Q$ is changed,

$$
v^{\mathrm{G}}(Q)=\frac{1}{2 M}\left\langle\nabla_{Q} \phi(q ; Q)\left|\hat{P}_{\perp}\right| \nabla_{Q} \phi(q ; Q)\right\rangle_{q} .
$$

Here $\hat{P}_{\perp}$ is a projector

$$
\hat{P}_{\perp}=1_{q}-|\phi(q ; Q)\rangle\left\langle\left.\phi(q ; Q)\right|_{q}\right.
$$

defined in the $q$ space that projects on the space orthogonal to $\phi(q ; Q) ; \hat{P}_{\perp}$ is necessary to make the geometric potential $v^{\mathrm{G}}$ gauge invariant, i.e., to make it independent of the choice of the phase $S(Q)$ in (5). The symbol $1_{q}$ represents the unity operator in $q$ space.

With these definitions, the choice of the phase $S$ for the wave function $\chi$ only affects the vector potential $A$. Choosing a different phase, i.e.,

$$
\begin{gathered}
\tilde{\chi}(Q)=e^{-i \tilde{S}(Q)} \chi(Q), \\
\tilde{\phi}(q ; Q)=e^{+i \tilde{S}(Q)} \phi(q ; Q),
\end{gathered}
$$

leaves all equations of the EF formalism unchanged provided the vector potential is changed as

$$
\tilde{A}(Q)=A(Q)+\nabla_{Q} \tilde{S}(Q) .
$$

The choice of the phase $S$ of $\chi$ is thus a choice of gauge and different gauges are considered below. Observables need to be independent of the choice of $S$. Such observables for $\mathscr{Q}$ are, e.g., the position expectation value

$$
\langle Q\rangle=\langle\chi|Q| \chi\rangle,
$$

the momentum expectation value

$$
\langle P\rangle=\left\langle\chi\left|-i \nabla_{Q}+A(Q)\right| \chi\right\rangle,
$$

or the expectation value of the kinetic energy

$$
\langle T\rangle=\left\langle\chi\left|\frac{\left[-i \nabla_{Q}+A(Q)\right]^{2}}{2 M}+v^{\mathrm{G}}(Q)\right| \chi\right\rangle,
$$

which are all expectation values of the particles of $\mathscr{Q}$ in the presence of the environment $\mathscr{E}$.

\section{EXCITED STATES AND NODES IN THE WAVE FUNCTION}

With the formalism for the EF we now study what the wave function $\chi_{j}$ of the quantum system $\mathscr{Q}$ looks like for an excited state $\psi_{j}$ of the supersystem $\mathscr{S}$. For this purpose, we use a simple $\mathscr{S}$ composed of two particles at $x_{1}$ and $x_{2}$ with masses

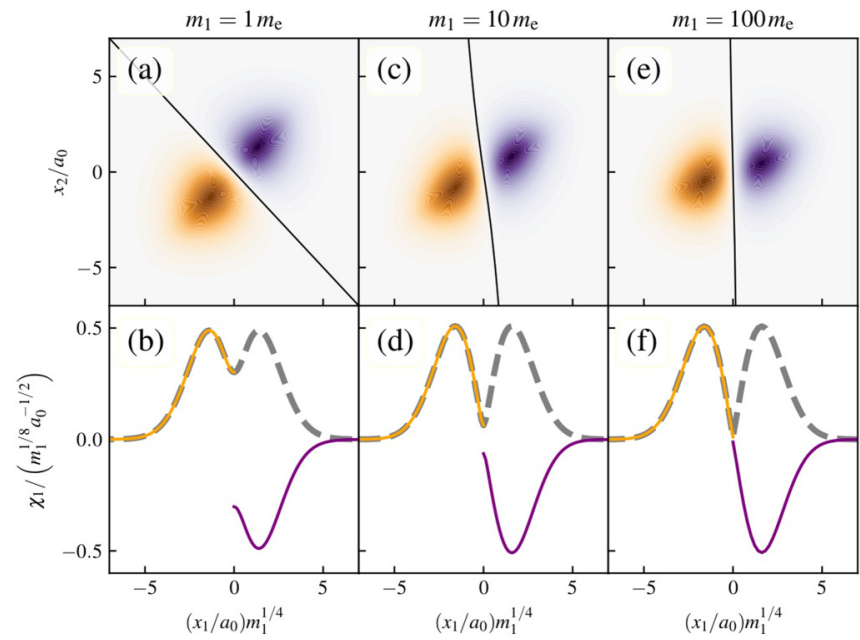

FIG. 2. (a), (c), and (e) Contour plot of the wave function $\psi_{1}\left(x_{1}, x_{2}\right)$ with colors indicating the sign and (b), (d), and (f) corresponding marginal wave function $\chi_{1}\left(x_{1}\right)$ for different values of the mass parameter $m_{1}$. The marginal wave function $\chi_{1}$ is shown in the even gauge (25) (gray dashed line) and in the odd gauge (26) with a sign change at $x_{1}=0$ (thin solid line). The coordinate $x_{1}$ is scaled with $m_{1}^{1 / 4}$ for better comparison, and $\chi_{1}$ is renormalized accordingly.

$m_{1}$ and $m_{2}$, respectively, that are both localized around the origin by a harmonic potential and that attract each other via a soft Coulomb potential (to avoid the Coulomb singularity in one dimension). The Schrödinger equation is

$$
\left(-\frac{\partial_{1}^{2}}{2 m_{1}}+H\left(x_{1}\right)\right) \psi_{j}\left(x_{1}, x_{2}\right)=E_{j} \psi_{j}\left(x_{1}, x_{2}\right),
$$

with the environment Hamiltonian

$$
H\left(x_{1}\right)=-\frac{\partial_{2}^{2}}{2 m_{2}}-\frac{1}{\sqrt{\left(x_{1}-x_{2}\right)^{2}+b}}+\left(\frac{x_{1}}{c_{1}}\right)^{2}+\left(\frac{x_{2}}{c_{2}}\right)^{2}
$$

and with parameters $b=0.5 a_{0}^{2}$ and $c_{1}=c_{2}=5 a_{0}$. For the particle at $x_{2}$, which will be the environment, we use the electron mass $m_{2}=m_{\mathrm{e}}$. If we chose $m_{1}=m_{\mathrm{e}}$, the model can be interpreted as positronium in a harmonic trap.

The first excited state $\psi_{1}\left(x_{1}, x_{2}\right)$ is antisymmetric with respect to the exchange of $x_{1}$ and $-x_{2}$ and thus has a node along $x_{1}=-x_{2}$. It is shown in Fig. 2(a). In Fig. 2(b) the marginal wave function

$$
\chi_{1}\left(x_{1}\right)=e^{-i S_{1}\left(x_{1}\right)} \sqrt{\rho_{1}\left(x_{1}\right)}
$$

with marginal density

$$
\rho_{1}\left(x_{1}\right)=\int_{-\infty}^{+\infty}\left|\psi_{1}\left(x_{1}, x_{2}\right)\right|^{2} d x_{2}
$$

is shown for the gauge

$$
\begin{aligned}
S_{1}\left(x_{1}\right) & =0 \\
\Rightarrow A\left(x_{1}\right) & =0
\end{aligned}
$$


and for the gauge

$$
\begin{aligned}
S_{1}\left(x_{1}\right) & =\pi \theta\left(x_{1}\right) \\
\Rightarrow A\left(x_{1}\right) & =\pi \delta\left(x_{1}\right) .
\end{aligned}
$$

The function $\theta(x)$ is the unit step function, with its derivative, the delta function $\delta(x)$. We call the gauge (25) the even gauge and the gauge (26) the odd gauge because $\chi_{1}$ is an even or odd function in these gauges. In the even gauge, $\chi_{1}$ is the positive square root of the marginal density and a continuous function. In the odd gauge, the sign of $\chi_{1}$ changes at $x_{1}=0$. As $\left|\chi_{1}(0)\right| \neq 0$, the function $\chi_{1}$ is discontinuous in this gauge. The effect of the $\delta$ function in the vector potential $A\left(x_{1}\right)$ is that it compensates for the discontinuity of $\chi_{1}$ in the Schrödinger equation and in the calculation of the gauge-invariant observables. From Fig. 2 it is clear that, due to the large jump of $\chi_{1}$ at $x_{1}=0$, there is very little reason to use the odd gauge.

If the mass parameter $m_{1}$ of the particle in $\mathscr{Q}$ is increased (relative to the mass of the particle in the environment), the nodal line of $\psi_{1}\left(x_{1}, x_{2}\right)$ becomes closer and closer to being perpendicular with respect to the $x_{1}$ axis. For the case $m_{1}=$ $100 m_{\mathrm{e}}$ the nodal line is almost perpendicular, as shown in Fig. 2(e). The marginal density $\rho_{1}\left(x_{1}\right)$ becomes very small at $x_{1}=0$ because the integration of $\left|\psi_{1}\right|^{2}$ along $x_{2}$ is now almost along the nodal line. The even gauge still yields a continuous marginal wave function $\chi_{1}$, but its slope in the region $x_{1} \approx 0$ looks almost discontinuous on the scale of the figure (which is the relevant scale of the problem), with a sharp turn at $x_{1}=0$. This has to be contrasted with the odd gauge: Although the function $\chi_{1}$ is still discontinuous at $x_{1}=0$, the fact that $\left|\chi_{1}\right|$ becomes very small leaves the impression that the odd gauge is the "natural" choice. If $\chi_{1}\left(x_{1}\right)$ was actually zero at $x_{1}=0$, it would be a continuous function in the odd gauge and its first derivative would be continuous as well.

Thus, for large $m_{1}$ and in the odd gauge, the wave function $\chi_{1}\left(x_{1}\right)$ of $\mathscr{Q}$ that corresponds to the first excited state of $\mathscr{S}$ also looks like an excited state of some potential. There is still a $\delta$ function appearing in the vector potential at $x_{1}=0$. However, in the Schrödinger equation and in the calculation of observables the vector potential is always multiplied by $\chi_{1}$ and $\left|\chi_{1}\left(x_{1}=0\right)\right|$ becomes smaller and smaller with increasing $m_{1}$. For $m_{1} \rightarrow \infty$, the nonzero vector potential thus becomes irrelevant.

The limit $m_{1} \rightarrow \infty$ corresponds to the BOA, which is also the limit where an excitation of $\mathscr{S}$ can be seen as an excitation of $\mathscr{Q}$ with no effect on its environment $\mathscr{E}$; the excitation of the two subsystems happens on different timescales. While $\chi_{1}$ in the odd gauge and for large $m_{1}$ looks like a first excited state, the corresponding conditional wave function of the environment looks like the ground-state wave function, as shown in Appendix A. Without invoking the BOA, however, each state $\psi_{j}$ of the supersystem $\mathscr{S}$ corresponds in principle not only to one marginal wave function $\chi_{j}$, but also to one conditional wave function $\phi_{j}$ and hence to one set of scalar potential $v_{j}$ and vector potential $A_{j}$. Thus, $\mathscr{Q}$ is not described by the eigenstates of only one Hamiltonian. Instead, all the $\chi_{j}$ are the ground states of their corresponding potentials $\left\{v_{j}, A_{j}\right\}$. In the following, we study the behavior of the potentials $v_{j}$ in the BOA limit.

\section{SPIKY POTENTIALS}

To show how the potentials $v_{j}$ corresponding to different states of the supersystem behave, we turn to a model that was already used in connection with the EF in [9]. This model represents some of the basic features of a symmetric coupled electron-proton transfer. We choose it here because it results in the well-known double-well potential for a tunneling dynamics (e.g., of the ammonia molecule) within the BOA and is thus a familiar system for most readers. Its Schrödinger equation is

$$
\left(-\frac{\partial_{R}^{2}}{2 M}+H(R)\right) \psi_{j}(R, x, y)=E_{j} \psi_{j}(R, x, y),
$$

with the electronic Hamiltonian

$$
H(R)=-\frac{\partial_{x}^{2}}{2}-\frac{\partial_{y}^{2}}{2}+V(R, x, y),
$$

where the potential

$$
\begin{aligned}
V= & -\frac{1}{\sqrt{x^{2}+y^{2}+\alpha_{2}}}+\left(\frac{R}{R_{0}}\right)^{4}+\frac{1}{\sqrt{R^{2}+\beta}} \\
& -\frac{1}{\sqrt{(R-x)^{2}+y^{2}+\alpha_{1}}}
\end{aligned}
$$

can be interpreted as that of a nucleus of mass $M$ located at $(X, Y)=(R, 0)$ that is allowed to move in the $R$ direction, an electron at $(x, y)$, and another nucleus clamped at $(X, Y)=$ $(0,0)$. A quartic potential is added to make the whole system bound. The parameters are $\alpha_{1}=0.5 a_{0}^{2}$ and $\alpha_{2}=\beta=4 a_{0}^{2}$, and we use a nuclear mass of only $M=30 m_{\mathrm{e}}$ to be able to clearly see the effect of the mass difference between the electron and nucleus.

Figure 3(a) shows the ground and first excited nuclear state of the system within the BOA. For this purpose, $v_{0}^{\mathrm{BO}}$ for the lowest eigenstate of the electronic Hamiltonian $H$ is determined by solving

$$
H(R) \phi_{j}^{\mathrm{BO}}(x, y ; R)=v_{j}^{\mathrm{BO}}(R) \phi_{j}^{\mathrm{BO}}(x, y ; R)
$$

for fixed values of $R$. The function $v_{0}^{\mathrm{BO}}(R)$ is a symmetric double-well potential along $R$. Then $v_{0}^{\mathrm{BO}}$ is used as the potential for the nucleus and its lowest two eigenstates $\chi_{0,0}^{\mathrm{BO}}$ and $\chi_{0,1}^{\mathrm{BO}}$ are determined by solving

$$
\left(-\frac{\partial_{R}^{2}}{2 M}+v_{0}^{\mathrm{BO}}(R)\right) \chi_{0, k}^{\mathrm{BO}}(R)=E_{0, k}^{\mathrm{BO}} \chi_{0, k}^{\mathrm{BO}}(R) .
$$

The nuclear states $\chi_{0,0}^{\mathrm{BO}}$ and $\chi_{0,1}^{\mathrm{BO}}$ form an almost degenerate tunneling doublet, with the energetically lower even eigenstate $\chi_{0,0}^{\mathrm{BO}}(R)$ and the energetically higher odd eigenstate $\chi_{0,1}^{\mathrm{BO}}(R)$. Thus, while the ground-state density $\left|\chi_{0,0}^{\mathrm{BO}}(R)\right|^{2}$ becomes small around $R=0$ but is always larger than zero, $\left|\chi_{0,1}^{\mathrm{BO}}(R)\right|^{2}$ is zero at $R=0$, as expected for the first excited state of the potential.

The situation is different when viewed in the EF formalism. Each eigenstate $\psi_{j}$ of the Schrödinger equation (27) corresponds to a nuclear wave function $\chi_{j}(R)$ but also to a potential $v_{j}(R)$. The nuclear wave functions $\chi_{j}(R)$ in this example are always the energetically lowest eigenstates of $v_{j}(R)$; hence they have no nodes. In Fig. 3(b) those eigenstates together 


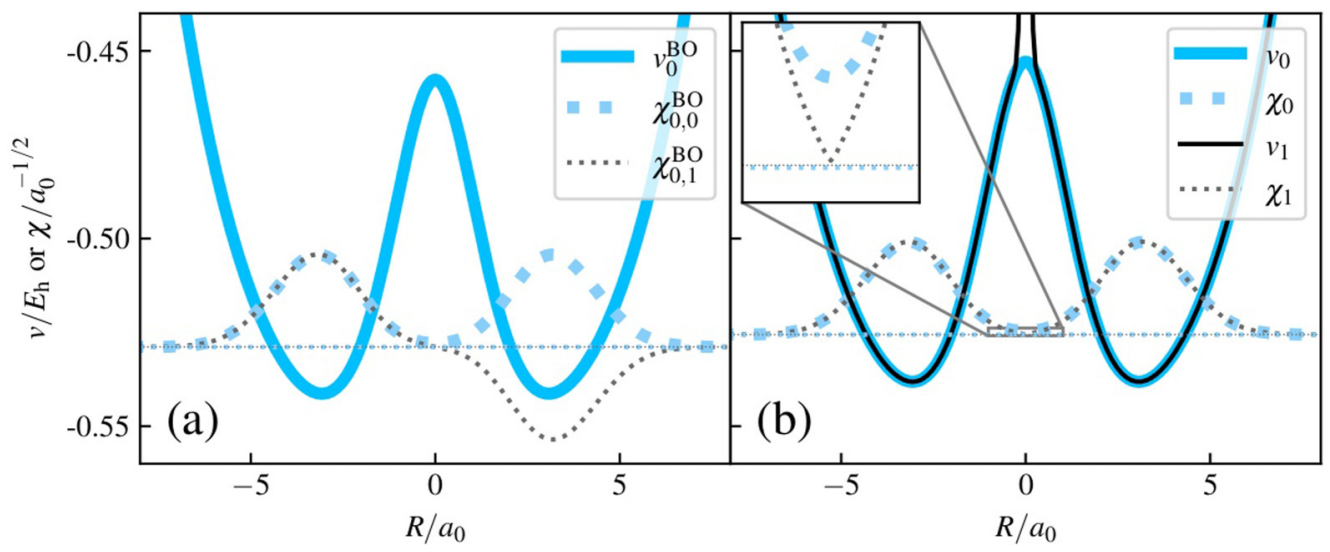

FIG. 3. Nuclear states of the electron-proton transfer model (27). (a) Lowest electronic Born-Oppenheimer surface $v_{0}^{\mathrm{BO}}(R)$ together with the two lowest Born-Oppenheimer nuclear wave functions $\chi_{0, j}^{\mathrm{BO}}$ (shifted to their eigenvalues $E_{0, j}^{\mathrm{BO}}$ ). (b) Exact factorization marginal potentials $v_{j}$ and marginal wave functions $\chi_{j}$ (shifted to their eigenvalues) of the two lowest eigenstates of (27).

with their potentials are shown for the gauge equivalent to (25), where $\chi_{j}(R)$ is positive (real valued) and $A(R)=0$. The nuclear ground state $\chi_{0}(R)$ and its EF potential $v_{0}(R)$ look very similar to $\chi_{0,0}^{\mathrm{BO}}(R)$ and $v_{0}^{\mathrm{BO}}(R)$, respectively. However, like $\chi_{0}(R)$, also $\chi_{1}(R)$ has no change of sign at $R=0$ in our chosen gauge, and the marginal density $\left|\chi_{1}(R)\right|^{2}$ is also larger than zero for $R=0$, albeit smaller than $\left|\chi_{0}(R)\right|^{2}$ in that region [see the inset of Fig. 3(b); $\chi_{1}$ becomes almost zero but does not reach it]. The corresponding EF potential $v_{1}(R)$ is mostly similar to $v_{0}^{\mathrm{BO}}(R)$, but markedly different around $R=0$ : There $v_{1}(R)$ has a narrow spike of finite height. This spike is the reason that the density of the first excited state $\left|\chi_{1}(R)\right|^{2}$ is smaller than that of the ground state $\left|\chi_{0}(R)\right|^{2}$ around $R=0$, although both are the ground states of otherwise similar potentials. If the mass of the nucleus is increased, the spike becomes narrower and higher and $\left|\chi_{1}(R=0)\right|^{2}$ becomes closer and closer to zero. However, the spikes are only spiky on the scale of the problem: Both the marginal wave functions and the potentials are continuous smooth functions, as would be visible if we would zoom in on them.

We thus see how the BOA picture emerges from the EF description in the limit of large nuclear mass: The potentials $v_{j}$ for different states $\psi_{j}$ of the supersystem become more and more equal with higher and higher mass of the marginal system, until the marginal states can be treated as different states of the same potential $v$. This is the limit where the environment $\mathscr{E}$ (here the electron) of the quantum system $\mathscr{Q}$ (here the nucleus) can be fully described with one potential. Thus, $\mathscr{Q}$ can be considered in the usual way as a quantum system with one Hamiltonian and its eigenstates.

\section{EIGENFUNCTIONS WITH A NODE}

In the EF, each state $\psi$ of the quantum supersystem $\mathscr{S}$ gives rise to a potential $v$ for the marginal system $\mathscr{Q}$. As seen in the examples of the two preceding sections, the marginal wave function $\chi$ typically has no nodes and is the ground state of the EF potential $v$, even though it may correspond to an excited state of $\mathscr{S}$. Only in the limit of the BOA can $\chi$ have nodes and be considered as an excited state itself. Naturally this raises the question if it is possible for the marginal wave function $\chi$ to have a node or nodes by construction or due to symmetry and if $\chi$ can then also be an excited state of $v$. The $\chi$ is usually nodeless because the condition to have an exact node is rather strong: The density $|\psi(Q ; q)|^{2}$ (which is a semidefinite-positive function) must be zero at the location $Q=Q_{0}$ of the node for all values of the coordinates $q$. For a general quantum system this is a very unlikely situation. However, such situations can be constructed explicitly.

Nodes in nonadiabatic eigenfunctions have been discussed in the literature. For example, in [32] a wave function

$$
\psi(R, q)=\chi_{1}^{\mathrm{BO}}(R) \phi_{1}^{\mathrm{BO}}(q ; R)+\chi_{2}^{\mathrm{BO}}(R) \phi_{2}^{\mathrm{BO}}(q ; R)
$$

was considered for a diatomic molecule with internuclear distance $R$, where $\phi_{j}^{\mathrm{BO}}$ is an eigenstate of the electronic Hamiltonian with electronic coordinates $q$. In the article it was concluded that whenever $\chi_{1}^{\mathrm{BO}}\left(R_{0}\right)=\chi_{2}^{\mathrm{BO}}\left(R_{0}\right)=0$ for some $R_{0}$, there is also a node in the marginal wave function $\chi\left(R_{0}\right)=0$ corresponding to $\psi(R, q)$. At these positions $R_{0}$, also singularities appear in the potential $v(R)$. Singularities and nodes are also shown in [32] for one example eigenstate. However, closer inspection and our own eigenvalue calculations for the same system reveal that the nodes of $\chi_{1}^{\mathrm{BO}}$ and $\chi_{2}^{\mathrm{BO}}$ do not appear at the same position $R$, only in vicinity of each other. Therefore, the marginal wave function $\chi(R)$ does not have nodes, only regions where the marginal density $|\chi(R)|^{2}$ becomes very close to zero, similar to the situation in Fig. 3(b). Hence, there are spikes of finite height in the corresponding potential $v(R)$, as also illustrated in another article on the same system [33]. Further details for our calculations of this system are given in Appendix B.

A second literature example is the articles [36,37], where marginal wave functions with nodes are constructed at submerged conical intersections of molecular systems. In this case the node is possible due to the degeneracy of the involved states and the symmetry of the model. However, we are somewhat skeptical regarding the transfer of the model of [36,37] to realistic molecules, for the following reasons. There are discussions of the exact static properties and of the dynamics through a conical intersection presented in [42,43], respectively, which show that at the location of a conical intersection there is nothing special happening, except if considered in the 
Born-Oppenheimer framework. Also, degeneracies of electronic wave functions in the Born-Oppenheimer framework need not correspond to degenerate states for the full electronnuclear problem. Finally, when treating the separation of coordinates including the center of mass and the rotational degrees of freedom fully correctly, we think that it is even less likely to find locations where the full wave function has a node along a set of coordinates (e.g., all electronic coordinates for a given nuclear configuration) such that the marginal wave function has a node there.

In the following, we present two methods on how to construct marginal wave functions with nodes, both also using degenerate states. These methods are given here to show that it is in principle possible to construct such marginal wave functions, but we do not claim (or assume) that they can be transferred to general high-dimensional interacting systems, like the nuclei and electrons of a molecule.

\section{A. Radially symmetric eigenfunctions}

In the first method, we use the radial symmetry of a two-dimensional problem and interpret it as a two-particle one-dimensional system. The Schrödinger equation for two particles with unit mass, constrained to move in one dimension, is

$$
\left(-\frac{\partial_{1}^{2}}{2}-\frac{\partial_{2}^{2}}{2}+V\left(x_{1}, x_{2}\right)\right) \psi\left(x_{1}, x_{2}\right)=E \psi\left(x_{1}, x_{2}\right) .
$$

If the potential is radially symmetric in the sense that

$$
V\left(x_{1}, x_{2}\right)=V(r),
$$

with $x_{1}=r \cos \varphi$ and $x_{2}=r \sin \varphi$, we can write the Hamiltonian in polar coordinates as

$$
\hat{H}=-\frac{\partial_{r}}{2 r}\left(r \partial_{r}\right)-\frac{1}{2 r^{2}} \partial_{\varphi}^{2}+V(r) .
$$

As the Hamiltonian commutes with the operator $\partial_{\varphi}^{2}$, both operators share common eigenfunctions. The eigenfunctions of the operator $\partial_{\varphi}^{2}$ are the eigenfunctions of the "angular momentum operator" $\hat{L}=-i \partial_{\varphi}$,

$$
\partial_{\varphi} e^{i l \varphi}=i l e^{i l \varphi} \Rightarrow \partial_{\varphi}^{2} e^{i l \varphi}=-l^{2} e^{i l \varphi},
$$

where $l \in \mathbb{Z}$. As the eigenspace of eigenfunctions of the operator $\partial_{\varphi}^{2}$ is doubly degenerated for $|l| \neq 0$, we have the freedom of choosing any linear combination of eigenfunctions $e^{+i|l| \varphi}$ and $e^{-i|l| \varphi}$. Thus, we can choose an eigenfunction with a nodal plane at $\varphi= \pm \pi / 2$, i.e., with a nodal plane at $x_{1}=0$, as

$$
\sin \left[|l|\left(\varphi-\frac{\pi}{2}\right)\right]=e^{+i|l| \varphi}-(-1)^{|l|} e^{-i|l| \varphi} .
$$

The general eigenstate of (33) is of the form

$$
\psi\left(x_{1}, x_{2}\right)=\psi_{\mathrm{r}}(r) \sin \left[|l|\left(\varphi-\frac{\pi}{2}\right)\right],
$$

where $|l| \in \mathbb{N}_{1}=\{1,2,3, \ldots\}$ and $\psi_{\mathrm{r}}(r)$ is a solution of the radial Schrödinger equation with the Hamiltonian

$$
\hat{H}_{\mathrm{r}}=-\frac{\partial_{r}}{2 r}\left(r \partial_{r}\right)+\frac{|l|^{2}}{2 r^{2}}+V(r) .
$$

By construction, the marginal wave function $\chi\left(x_{1}\right)$ corresponding to the wave function $\psi\left(x_{1}, x_{2}\right)$ of (38) has a node at $x_{1}=0$.

This method works for any choice of the potential $V(r)$, but it is instructive to show it for a concrete example. We choose the Coulomb potential

$$
V\left(x_{1}, x_{2}\right)=-\frac{1}{\sqrt{x_{1}^{2}+x_{2}^{2}}} ;
$$

hence we describe a one-dimensional electron-positron system (positronium). The two-dimensional solutions are eigenstates $|n, l\rangle$ with energy quantum number $n \in\{1,2,3, \ldots\}$ and angular momentum quantum number $l \in\{-(n-$ $1), \ldots,-1,0,1, \ldots, n-1\}$ (the explicit form of the functions can be found in [44]),

$$
\begin{gathered}
\hat{H}|n, l\rangle=E_{n}|n, l\rangle, \quad E_{n}=-\frac{1}{2\left(n-\frac{1}{2}\right)^{2}}, \\
\hat{L}|n, l\rangle=l|n, l\rangle .
\end{gathered}
$$

Using the linear combination (37), we can construct the desired wave functions for $n \in\{2,3, \ldots\}$ and $|l| \in$ $\{1,2, \ldots, n-1\}$ as

$$
\psi_{n,|l|}\left(x_{1}, x_{2}\right)=\frac{1}{\sqrt{2}}\left[|n,+| l|\rangle-(-1)^{|l|}|n,-| l|\rangle\right] .
$$

Two of these wave functions are

$$
\begin{aligned}
& \psi_{2,1}\left(x_{1}, x_{2}\right)=\frac{8}{9} \sqrt{\frac{2}{3 \pi}} x_{1} e^{-(2 / 3) \sqrt{x_{1}^{2}+x_{2}^{2}}}, \\
& \psi_{3,2}\left(x_{1}, x_{2}\right)=\frac{32}{125} \sqrt{\frac{2}{15 \pi}} x_{1} x_{2} e^{-(2 / 5) \sqrt{x_{1}^{2}+x_{2}^{2}}},
\end{aligned}
$$

which correspond to the marginal densities

$$
\begin{aligned}
\left|\chi_{2,1}\left(x_{1}\right)\right|^{2} & =\frac{256}{243 \pi} x_{1}^{3} K_{1}\left(\frac{4}{3}\left|x_{1}\right|\right), \\
\left|\chi_{3,2}\left(x_{1}\right)\right|^{2} & =\frac{1024}{46875 \pi} x_{1}^{4} K_{2}\left(\frac{4}{5}\left|x_{1}\right|\right),
\end{aligned}
$$

where $K_{n}(x)$ are the modified Bessel functions of the second kind. We can choose an even or odd gauge for the marginal wave functions, like we did in Sec. III. The choice of odd marginal wave functions and the corresponding EF potentials is shown in Fig. 4. For states $|l|=1$ the EF potential $v\left(x_{1}\right)$ diverges at the origin approximately as $\ln \left|x_{1}\right|$. Although the potential is unbounded, the spectrum is bounded as the logarithm is slowly divergent at the origin. For $|l|>1, v\left(x_{1}\right)$ is finite and bounded and so is the spectrum. As we can see in Fig. 4, the marginal wave functions $\chi_{n,|l|}$ correspond to the first excited state of the EF potential.

If we chose an even marginal wave function instead, it has a cusp at the origin. Then an additional term of the form $\delta\left(x_{1}\right) /\left|x_{1}\right|$ appears in $v(x)$ and $\chi_{n,|l|}$ corresponds again to the ground state of this potential.

\section{B. Factorizable wave functions}

The second method to construct marginal wave functions with a node is based on the idea of factorizable eigenfunctions 


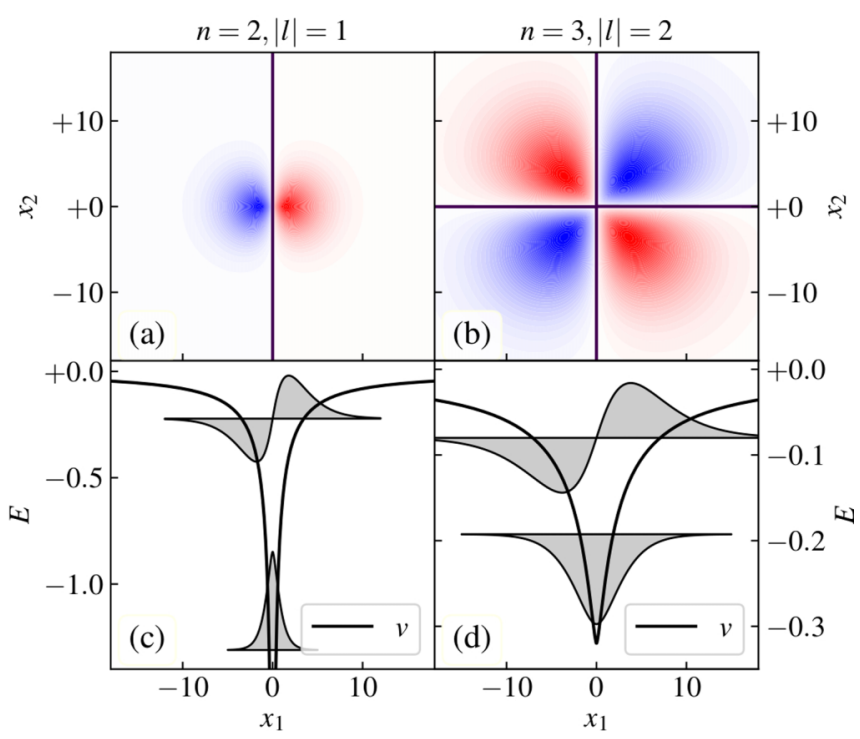

FIG. 4. (a) and (b) Two wave functions $\psi_{n,|l|}\left(x_{1}, x_{2}\right)$ defined by (43) for the one-dimensional positronium model, with nodes indicated by black lines and the color of the contour plot indicating the sign of the wave function. (c) and (d) Corresponding EF potential $v\left(x_{1}\right)$ as well as the ground and the first excited state of $v\left(x_{1}\right)$ for $\chi_{n,|l|}\left(x_{1}\right)$ for the densities given in (45). The sign of $\chi_{n,|l|}$ was chosen such that it is an odd function. The first excited state of $v\left(x_{1}\right)$ corresponds to $\chi_{n,|l|}\left(x_{1}\right)$.

that have a node at same position. We assume a system of two particles of mass $m=1$, whose Hamiltonian is separable as

$$
\hat{H}\left(x_{1}, x_{2}\right)=\hat{h}\left(x_{1}\right)+\hat{h}\left(x_{2}\right) \text {, }
$$

where $\hat{h}$ is a one-particle Hamiltonian

$$
\hat{h}\left(x_{1}\right)=-\frac{\partial_{1}^{2}}{2}+V\left(x_{1}\right)
$$

From the eigenstates of the single-particle Hamiltonian $\hat{h}$,

$$
\hat{h} \xi_{n}\left(x_{1}\right)=E_{n} \xi_{n}\left(x_{1}\right)
$$

we construct the symmetrized states

$$
\psi_{m, n}^{ \pm}\left(x_{1}, x_{2}\right)=\frac{1}{\sqrt{2}}\left[\xi_{m}\left(x_{1}\right) \xi_{n}\left(x_{2}\right) \pm \xi_{n}\left(x_{1}\right) \xi_{m}\left(x_{2}\right)\right] .
$$

If $\xi_{m}$ and $\xi_{n}$ share a node at one position, this node will translate to the marginal wave function $\chi^{ \pm}$corresponding to $\psi^{ \pm}$. One way how to accomplish this is by choosing odd eigenfunctions of the symmetric one-particle potential $v$. For demonstration purposes we choose a harmonic potential $V\left(x_{1}\right)$ with eigenstates $\xi_{n}$. The energetically lowest states of this construction are

$$
\begin{gathered}
\psi_{1,3}^{+}\left(x_{1}, x_{2}\right)=\frac{2}{\sqrt{3 \pi}} x_{1} x_{2}\left(x_{2}^{2}+x_{1}^{2}-3\right) e^{-x_{1}^{2} / 2-x_{2}^{2} / 2}, \\
\psi_{1,3}^{-}\left(x_{1}, x_{2}\right)=\frac{2}{\sqrt{3 \pi}} x_{1} x_{2}\left(x_{2}^{2}-x_{1}^{2}\right) e^{-x_{1}^{2} / 2-x_{2}^{2} / 2} .
\end{gathered}
$$

Due to the orthogonality of one-particle eigenstates, the corresponding marginal densities as well as the EF potentials are the same for $\psi_{1,3}^{+}$and $\psi_{1,3}^{-}$,

$$
\begin{gathered}
\left|\chi^{ \pm}\left(x_{1}\right)\right|^{2}=\frac{1}{6 \sqrt{\pi}}\left(15 x_{1}^{2}-12 x_{1}^{4}+4 x_{1}^{6}\right) e^{-x_{1}^{2}}, \\
v^{ \pm}\left(x_{1}\right)=5+\frac{-1215+2553 x_{1}^{2}-2112 x_{1}^{4}+936 x_{1}^{6}-208 x_{1}^{8}+16 x_{1}^{10}}{2\left(15-12 x_{1}^{2}+4 x_{1}^{4}\right)^{2}}+\frac{\delta\left(x_{1}\right)}{\left|x_{1}\right|} .
\end{gathered}
$$

As in the last example, the presence of the term $\delta\left(x_{1}\right) /\left|x_{1}\right|$ depends on the choice of sign of the marginal wave function $\chi^{ \pm}\left(x_{1}\right)$. Figure 5 shows $\chi^{ \pm}$for the choice where it changes sign at $x_{1}=0, v^{ \pm}$has no spike, and $\chi^{ \pm}$also corresponds to the first excited state of this potential.

We remark that, coincidentally, the choice of the potential $V\left(x_{1}\right)$ as the harmonic potential leads also to a radially symmetric two-particle potential, as in the first method. However, the second method for constructing marginal wave functions with a node works for any choice of the one-particle potential $V\left(x_{1}\right)$ and does not depend on a radial symmetry of the problem. The harmonic potential is also the only case where the two methods coincide.

\section{DETERMINING NODES OF EXCITED STATES FROM GROUND-STATE CALCULATIONS}

Our explicit consideration of the quantum environment $\mathscr{E}$ of the quantum system $\mathscr{Q}$ showed that there is a correspondence between excited states of a spikeless potential $v=v_{0}$ and the ground state of a similar but spiky potential. In this section we use the correspondence for one-dimensional systems to determine the location of nodes of an excited state of $v_{0}$ from ground-state calculations by using a modified potential.

For this purpose, we define the Schrödinger equation

$$
\left(-\frac{\nabla_{Q}^{2}}{2 M}+v_{j}\left(Q ; \boldsymbol{Q}_{j}\right)\right) \chi_{j, k}\left(Q ; \boldsymbol{Q}_{j}\right)=E_{j, k}\left(\boldsymbol{Q}_{j}\right) \chi_{j, k}\left(Q ; \boldsymbol{Q}_{j}\right)
$$

with some set of parameters $\boldsymbol{Q}_{j}$ that denote the position of spikes. The potential $v_{0}$ is the spikeless potential and thus $\boldsymbol{Q}_{0}$ is an empty set. We assume that $Q$ is one dimensional, although some parts of the following discussion are also applicable for higher-dimensional problems.

In general, the $j$ th excited state $\chi_{0 j}$ of $v_{0}$ is the ground state of the potential

$$
v_{j}\left(Q ; \boldsymbol{Q}_{j}\right)=v_{0}(Q)+\sum_{k=1}^{j} d\left(Q_{j, k}\right)
$$




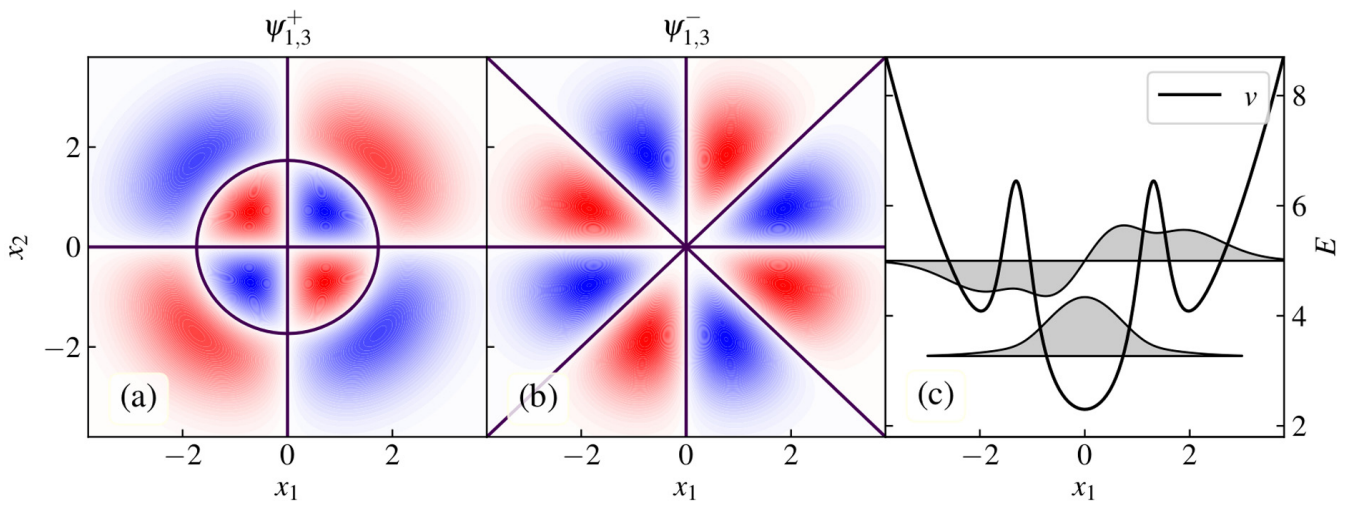

FIG. 5. (a) and (b) Wave functions $\psi_{1,3}^{ \pm}\left(x_{1}, x_{2}\right)$ of (50) and (51) (contour plots, where the colors indicate the sign and the lines are the nodal lines). (c) Plot of $v^{ \pm}$given in (53), without the $\delta\left(x_{1}\right) /\left|x_{1}\right|$ term, and its two lowest eigenstates. The $\chi^{ \pm}$of (52) corresponds to the first excited state of this potential if its sign is chosen such that it changes at $x_{1}=0$.

where $d\left(Q_{j, k}\right)$ are spikes at the $j$ configurations $Q_{j, k}$ that coincide with the location of the nodes of $\chi_{j}$. The parameter vectors are $\boldsymbol{Q}_{j}=\left(Q_{j, 1}, Q_{j, 2}, \ldots, Q_{j, j}\right)$. Although we consider only one-dimensional examples for $\mathscr{Q}$, this statement is true also for higher dimensions provided the real-valued eigenstates are chosen in the case of a degeneracy of states. In higher dimensions, each $Q_{j, k}$ is a nodal (hyper)surface, $j$ is the label of the energy eigenvalue counting degenerate states only once (because they have the same number of nodal surfaces), and each state of a set of degenerate states has its own $v_{j}$ with different $\boldsymbol{Q}_{j}$ (which would lead to another index to account for that).

We can use the potentials defined in (55) to calculate any excited state as the ground state of some $v_{j}$. Figure 6 exemplifies the basic idea with the model of a harmonic oscillator. Figure 6(a) shows the two lowest eigenstates of the harmonic oscillator potential $v_{0}(x)=\frac{x^{2}}{2}$. By symmetry, the first excited state must have a node at $x=0$; hence it can be computed as the ground state of a potential $v_{1}$ that is equal to $v_{0}$ except at $x=0$, where we add a spike by hand. For the numerical calculation we set $v_{1}(0)=10^{12} E_{\mathrm{h}}$ on the discrete $x$ grid, which provides the state shown in Fig. 6(b) as the ground state. Figure 6(c) shows the ground state of the half-harmonic-oscillator potential where the potential is set to
$10^{12} E_{\mathrm{h}}$ for all grid points $x \geqslant 0$, for comparison. There is no difference between the states and their eigenvalues within the numerical accuracy.

For the first excited state of the harmonic oscillator the position of the node is given by symmetry. In general, however, the locations $\boldsymbol{Q}_{j}$ of the nodes are not known and the potential $v_{j}$ of (55) cannot be set up immediately. However, there are two conditions for the values of $\boldsymbol{Q}_{j}$.

The first condition is that the energy of the ground state computed in each of the regions separated by the spikes at $\boldsymbol{Q}_{j}$ is the same. For example, in Fig. 6 we can move the spike and compute the eigensystem on left and right sides of the spike separately. The correct location of the spike is where the two energies match. This condition also holds for higher-dimensional problems where the regions are separated by hypersurfaces $\boldsymbol{Q}_{j}$.

The second condition for the values of $\boldsymbol{Q}_{j}$ is that the nodes of the $j$ th eigenstate $\chi_{0, j}$ of $v_{0}$ are at the positions where the energy $E_{i, j-i}\left(\boldsymbol{Q}_{j}\right)$ of the $(j-i)$ th eigenstate $\chi_{i, j-i}$ of $v_{i}(i \leqslant j)$ has its maxima as a function of the spike positions $\boldsymbol{Q}_{j}$. For definiteness, we call this condition the maxnode condition in the following, and it is only considered for one-dimensional problems. In the following, we illustrate it with an example. A
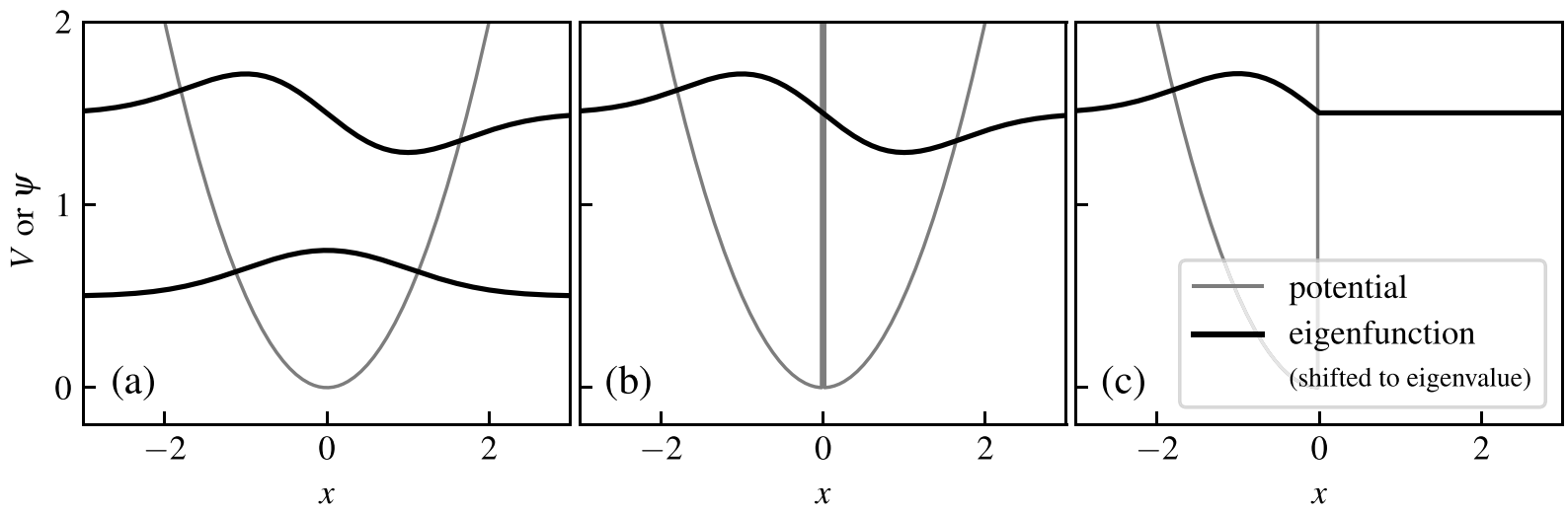

FIG. 6. (a) The two lowest eigenstates of a harmonic oscillator potential, (b) the lowest eigenstate of a harmonic oscillator potential with a large spike at $x=0$, and (c) the lowest eigenstate of a harmonic oscillator half-potential, shifted to their eigenvalues and shown together with their potentials. 

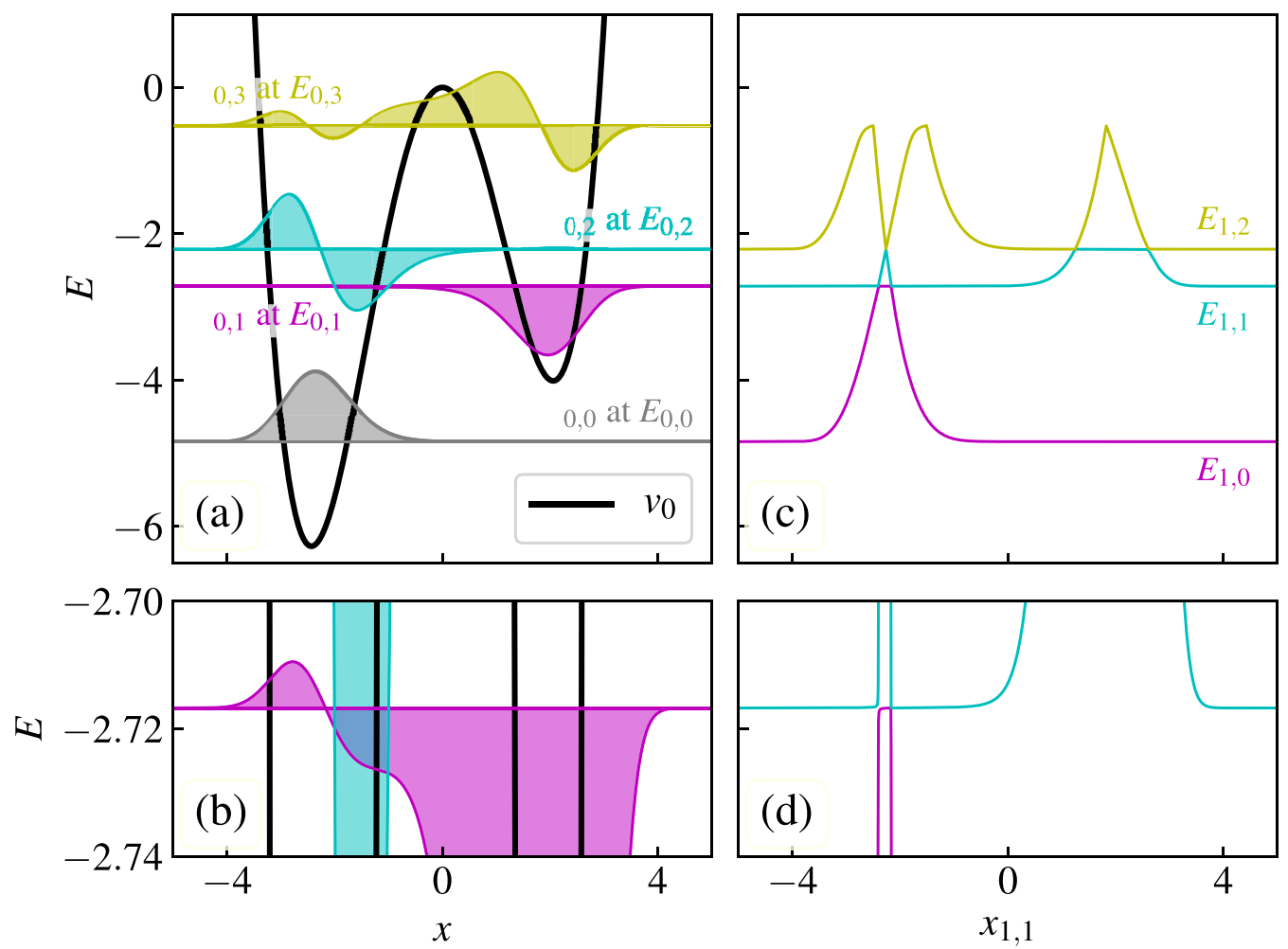

FIG. 7. (a) Potential $v_{0}$ of (56) shown together with its four lowest eigenfunctions (filled areas, shifted to their eigenvalues). (b) Same as (a) but for a narrow energy region to show the node of the first excited state of $v_{0}$. (c) Energy $E_{1, k}$ of the three lowest eigenstates of $v_{1}$ of (58) depending on the position of the spike at $x_{1,1}$. (d) Same as (c) but for the energetic region of (b). We note that $E_{1,0}$ has only one maximum and $E_{1,1}$ has only two maxima, even though the regions close to the maxima can become very flat as a function of $x_{1,1}$.

proof of the maxnode condition for one-dimensional problems is given in Appendix C.

In Fig. 7(a) the potential

$$
v_{0}(x)=0.2 x^{4}+0.1 x^{3}-2 x^{2}
$$

and its four lowest states $\chi_{0, j}$ (shifted to their corresponding eigenvalues $E_{0, j}$ ) are shown, determined from

$$
\left(-\frac{\partial_{x}^{2}}{2}+v_{0}(x)\right) \chi_{0, j}(x)=E_{0, j} \chi_{0, j}(x) .
$$

The first excited state has a node, as is visible in the detailed view of the energy region around the node, shown in Fig. 7(b). Figures $7(\mathrm{c})$ and $7(\mathrm{~d})$ show the energy $E_{1, k}$ of the three lowest states of

$$
v_{1}\left(x ; x_{1,1}\right)= \begin{cases}v_{0}(x) & \text { if } x \neq x_{1,1} \\ \infty & \text { if } x=x_{1,1}\end{cases}
$$

as a function of the location $x_{1,1}$ of the spike. In the numerical calculation, $\infty$ is replaced with $10^{12} E_{\mathrm{h}}$, this spike is moved along the $x$ grid, and an eigenvalue equation is solved for each position of the spike. As can be seen from the figure, the location of the node of the first excited state $\chi_{0,1}$ of $v_{0}$ is where the energy $E_{1,0}\left(x_{1,1}\right)$ of the ground state $\chi_{1,0}$ of $v_{1}$ is maximal as a function of the spike position $x_{1,1}$; Fig. 7(d) shows the relevant energetic region. Similarly, the two nodes of the second excited state $\chi_{0,2}$ of $v_{0}$ are where the energy $E_{1,1}\left(x_{1,1}\right)$ of the first excited state $\chi_{1,1}$ of $v_{1}$ has its maxima, and the three nodes of the third excited state $\chi_{0,3}$ of $v_{0}$ are where the energy $E_{1,2}\left(x_{1,1}\right)$ of the second excited state $\chi_{1,2}$ of $v_{1}$ has its maxima. In general, the nodes of the $j$ th eigenstate of $v_{0}$ are at the positions where the energy $E_{1, j-1}\left(x_{1,1}\right)$ of the $(j-1)$ th eigenstate of $v_{1}$ is maximal as a function of the spike position $x_{1,1}$.

The nodes of $\chi_{0,2}$ are also at the position where the ground state $\chi_{2,0}$ of a potential with two spikes,

$$
v_{2}\left(x ;\left(x_{2,1}, x_{2,2}\right)\right)= \begin{cases}v_{0}(x) & \text { if } x \neq x_{2,1}, x_{2,2} \\ \infty & \text { if } x=x_{2,1}, x_{2,2}\end{cases}
$$

has its maximum energy as a function of the position of the two spikes (not shown). In general, the nodes of the $j$ th eigenstate of $v_{0}$ are where the ground state of the potential $v_{j}$ with $j$ spikes has its maximum energy as a function of the spike positions. If we combine these observations, we have the maxnode condition.

Based on the maxnode condition, it is straightforward to propose an algorithm for one-dimensional problems to find the nodes of the $j$ th excited state as the ground state of $v_{0}$ with an additional $j$ spikes. First, $j$ spikes are added at random positions. Second, the gradient of the ground-state energy with respect to a change of the position of the spikes is calculated. Third, the spikes are shifted in the direction of the gradient. This procedure is repeated until the maximum energy of the ground state is found. The location of the spikes is then the location of the nodes of the $j$ th excited state of $v_{0}$.

For our model, there are some small gradients that make a numerical implementation difficult. The gradients are small in regions where there is a node but a very small amplitude of the wave function because of the asymmetric double-well 

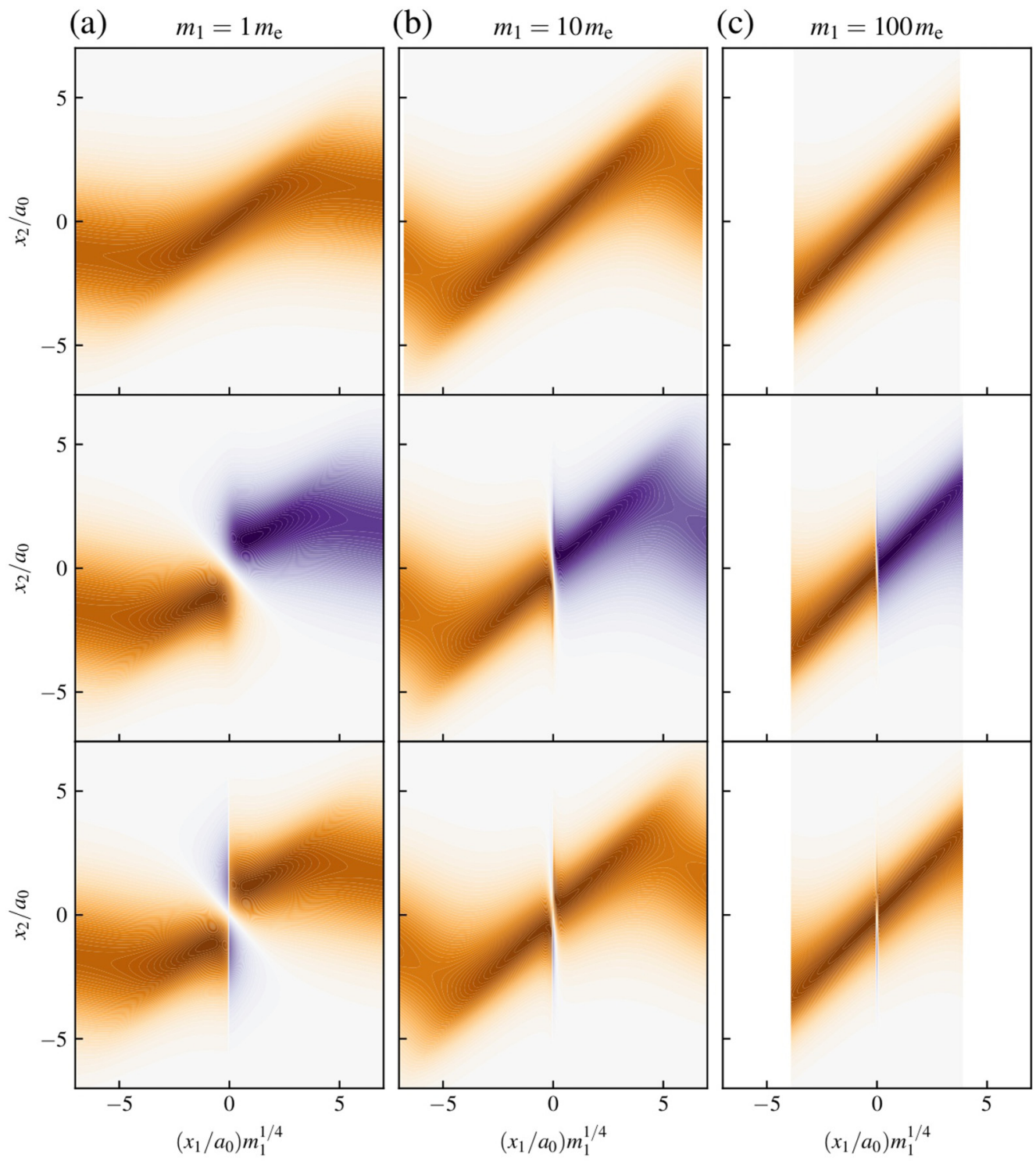

FIG. 8. Conditional wave functions (a) $\phi_{0}\left(x_{2} ; x_{1}\right)$, (b) $\phi_{1}\left(x_{2} ; x_{1}\right)$ for gauge (25), and (c) $\phi_{1}\left(x_{2} ; x_{1}\right)$ for gauge (26) for the ground state and for the first excited state of the model of two attracting particles in a harmonic trap discussed in Sec. III, respectively. The three columns correspond to the different masses $m_{1}$ of the particle at $x_{1}\left(m_{2}=1 m_{\mathrm{e}}\right)$.

structure. Hence, those small gradients are less of a problem if the model does not exhibit such states or if the evaluation of nodes in regions where there is almost no amplitude is not relevant.

Before concluding this section, we note a peculiarity of the energies $E_{1, k}\left(x_{1,1}\right)$ shown in Fig. 7(c): As those energies are obtained by solving (54) with the position $x_{1,1}$ being a parameter, the problem is similar to the Born-Oppenheimer calculation of a potential energy surface. Hence, $E_{1, k}\left(x_{1,1}\right)$ are "adiabatic surfaces" that are ordered according to their energetic numbering. Corresponding diabatic surfaces can be determined by continuing the functions along a coarsegrained slope on the scale of Fig. 7(c) [and not on the true slope given in Fig. 7(d)]. Such diabatic surfaces are shown in Appendix D. In this one-dimensional example there are no exact crossings of the surfaces, only avoided crossings. For higher-dimensional problems these surfaces can cross and the equivalent of conical intersections may appear.

\section{CONCLUSION}

Quantum systems are always open systems interacting with a quantum environment. While the typical approach to study open quantum systems is based on density matrix theory [29], already a seemingly closed quantum system with a Hamiltonian that contains potentials can be viewed as an open quantum system because the potentials encode an interaction with an unspecified environment.

Our first goal with this article was to illustrate how the EF describes a system and its environment. Using the EF, we studied how potentials emerge from explicit consideration of the quantum environment of a quantum system. We have shown that an exact treatment of the quantum system $\mathscr{Q}$ in its environment $\mathscr{E}$ is in general in terms of many potentials $v_{j}(Q)$ with their ground states $\chi_{j}(Q)$. In the limit of the BOA these potentials all become similar, except for localized finite spikes, and $\mathscr{Q}$ can approximately be described by a spikeless 


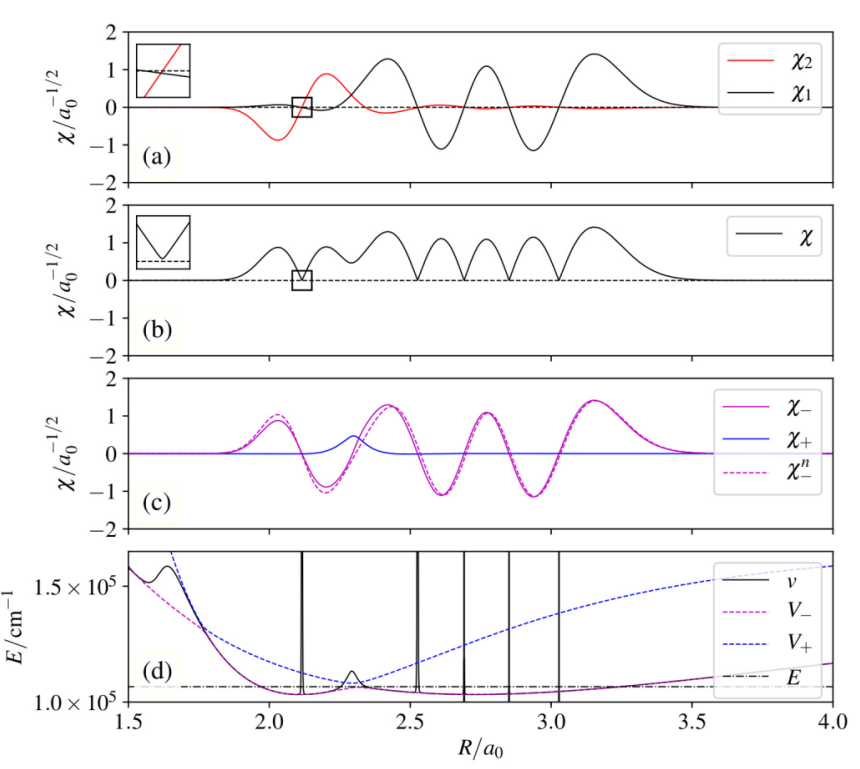

FIG. 9. (a) The two states $\chi_{1}$ and $\chi_{2}$ for the seventh excited state of the coupled Schrödinger equation (B1). (b) Corresponding

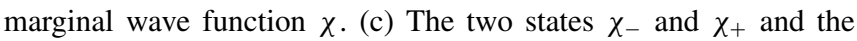
$(n=7)$ th eigenfunction $\chi_{-}^{n}$ for the potential $V_{-}$. (d) The EF potential $v$, the two adiabatic potentials $V_{-}$and $V_{+}$, and the eigenenergy $E$.

potential and its eigenstates. Excitations of the supersystem $\mathscr{S}$ composed of $\mathscr{Q}$ and $\mathscr{E}$ are then excitations of $\mathscr{Q}$ alone, with $\mathscr{E}$ being unaffected. In this way, the usual description of a (closed) quantum system in terms of a potential and its eigenstates is recovered.

Our second goal was to study what happens when $\chi_{j}(Q)$ has exact nodes. While the states $\chi_{j}(Q)$ of $\mathscr{Q}$ that follow from the EF are typically nodeless even if they correspond to excited states of $\mathscr{S}$, we also constructed situations where $\chi_{j}(Q)$ has exact nodes. Then $\chi_{j}(Q)$ can be viewed both as the ground state of a spiky potential or, more naturally, as the excited state of a spikeless potential.

Motivated by this insight, we have also achieved our third goal: For one-dimensional systems, we presented a method for finding the nodes of excited states based on ground-state calculations using spiky potentials. The idea on which our method is based may be interesting for quantum Monte Carlo calculations of fermionic problems. There the fixed-node variational principle is often used: The energy minimization of a trial wave function with its nodes restricted to the position of the nodes of some excited state yields exactly that excited state, as proven in [45]. As even the ground-state wave function of a many-fermion system is a highly excited state of the corresponding Hamiltonian without symmetry constraints, the function is strongly oscillating and evaluation of integrals (expectation values) is numerically difficult. A remedy for this so-called fermionic sign problem in quantum Monte Carlo methods is a fixed-node approximation [38] based on the fixed-node variational principle.

However, the fixed-node variational principle does only tell the location of the nodes. By using that spikes in the potential are equivalent to constraints on the position of the nodes, we have shown how the position of nodes can be obtained by an algorithm that shifts the spikes along the energy gradient obtained from a ground-state calculation, for one-dimensional systems. So far, we have not found a generalization of our method for higher dimensions, and we also think that further constraints may be needed in addition to the maxnode condition to find nodal surfaces in a similar way. Thus, it can be interesting and potentially rewarding to study how our insights can be applied to the computation of properties of higher-dimensional systems.

\section{ACKNOWLEDGMENT}

This research was supported by an Ambizione grant of the Swiss National Science Foundation.

\section{APPENDIX A: CONDITIONAL WAVE FUNCTION FOR THE MODEL OF SEC. III}

In Sec. III the first excited state $\psi_{1}\left(x_{1}, x_{2}\right)$ of the model of two attracting particles in a harmonic trap and its marginal wave function $\chi_{1}\left(x_{1}\right)$ was discussed. Figure 8 shows the conditional wave functions $\phi_{0}\left(x_{2} ; x_{1}\right)$ and $\phi_{1}\left(x_{2} ; x_{1}\right)$ for the ground state and the first excited state of the two-particle model, respectively. Here $\phi_{j}\left(x_{2} ; x_{1}\right)$ is the wave function of the environment $\mathscr{E}$ (the particle at $x_{2}$ ) for a given configuration $x_{1}$ of the particle in the quantum system $\mathscr{Q}$.

For the first excited state, $\phi_{1}\left(x_{2} ; x_{1}\right)$ is shown for the two gauges (25) [Fig. 8(b), where there is no sign change in the marginal wave function $\chi_{1}$ ] and (26) [Fig. 8(c), where there is a sign change in the marginal wave function $\left.\chi_{1}\right]$. With increasing mass $m_{1}$ of the particle in $\mathscr{Q}$, the state $\phi_{1}\left(x_{2} ; x_{1}\right)$ of the environment becomes very similar to that of the ground state, $\phi_{0}\left(x_{2} ; x_{1}\right)$, shown in Fig. 8(a). Like for $\chi_{1}$, we also find for $\phi_{1}$ that the gauge (25) is suitable for small masses $m_{1}$, while the gauge (26) becomes the natural gauge for larger $m_{1}$. For larger $m_{1}$, we can thus interpret the first excited state $\psi_{1}\left(x_{1}, x_{2}\right)$ as an excitation of $\mathscr{Q}$ alone, with the environment $\mathscr{E}$ staying in its ground state.

\section{APPENDIX B: ANALYSIS OF A NONADIABATIC EIGENFUNCTION}

In Sec. $\mathrm{V}$ we mentioned a literature example of nonadiabatic eigenfunctions that seem to have nodes but actually do not have them. From the ansatz (32), the problem is described with two coupled differential equations

$\left[\left(\begin{array}{cc}T_{\mathrm{N}} & 0 \\ 0 & T_{\mathrm{N}}\end{array}\right)+\left(\begin{array}{cc}V_{1}(R) & \gamma(R) \\ \gamma(R) & V_{2}(R)\end{array}\right)\right]\left(\begin{array}{c}\chi_{1}^{\mathrm{BO}}(R) \\ \chi_{2}^{\mathrm{BO}}(R)\end{array}\right)=E\left(\begin{array}{l}\chi_{1}^{\mathrm{BO}}(R) \\ \chi_{2}^{\mathrm{BO}}(R)\end{array}\right)$,

where $T_{\mathrm{N}}=-\frac{1}{2 M} \partial_{R}^{2}$ is the kinetic energy with reduced nuclear mass $M$. We used data from [46] to construct the Morse potential models of $V_{1}(R)$ and $V_{2}(R)$. As in [32], we use a constant coupling term $\gamma(R)=\gamma=890 \mathrm{~cm}^{-1}$ and, using QMSTUNFTI [39], we calculated the first 20 coupled eigenfunctions. Our calculated eigenenergies and occupation numbers for each state correspond to those in [32]. We demonstrate our results for the same state (the seventh excited state $v=4, b^{\prime}$ ) as in this article. Close inspection shows that the nodes of $\chi_{1}^{\mathrm{BO}}$ and $\chi_{2}^{\mathrm{BO}}$ do not appear at the same position $R$, only in the vicinity of each other, as can be seen in Fig. 9(a). The marginal wave 

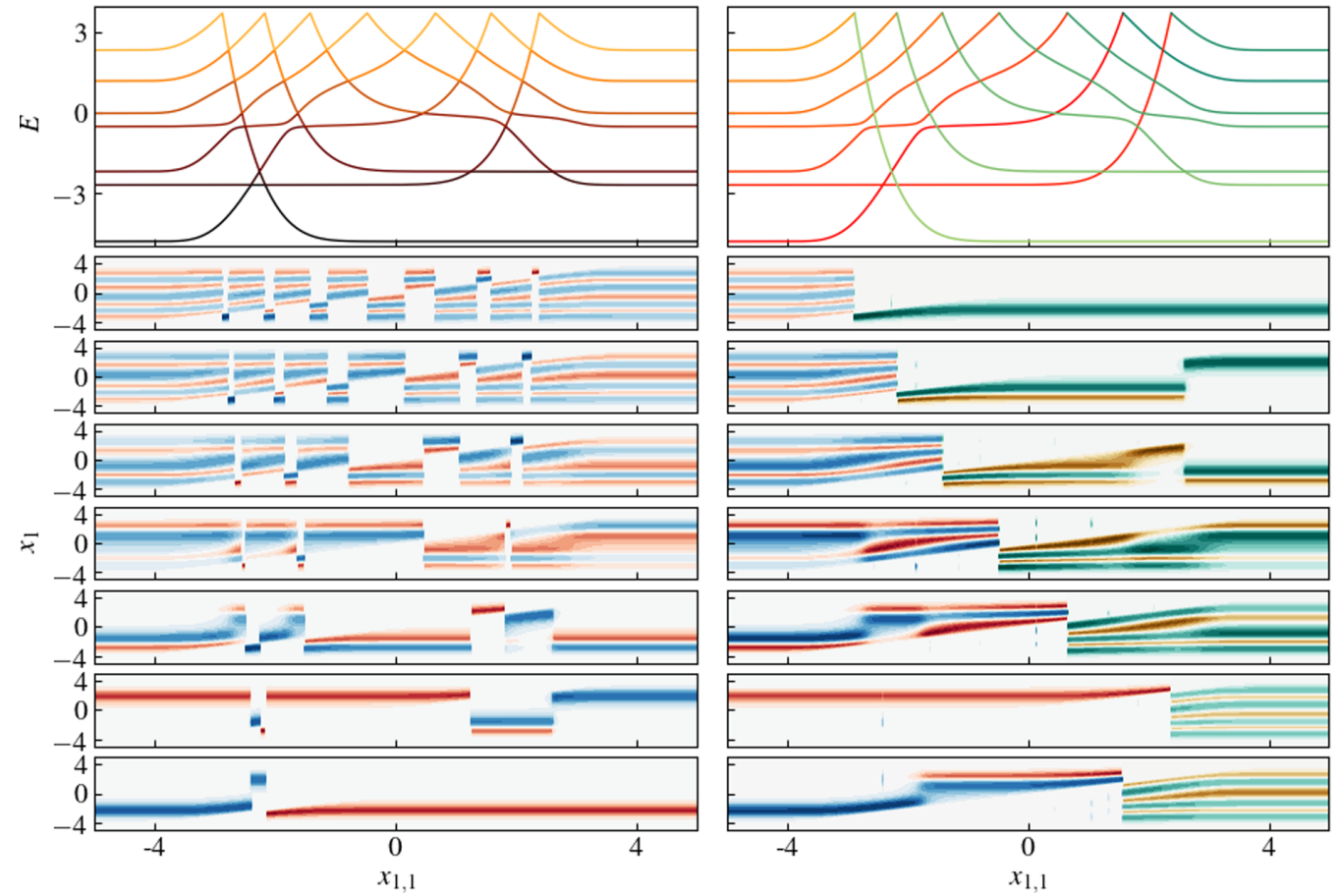

FIG. 10. Shown on top are the energies $E_{1, k}\left(x_{1,1}\right)$ for the seven lowest states of the potential $v_{1}$ given in (58) with a spike at $x_{1,1}$, sorted energetically (left, adiabatic) and according to linear extrapolation (right, diabatic). On the bottom are the corresponding wave functions $\chi_{1, k}\left(x_{1} ; x_{1,1}\right)$ with energy increasing from the bottom to the top panel, shown as a contour plot, corresponding to the adiabatic ordering (left) and diabatic ordering (right).

function $\chi$ gets close to zero, but never reaches zero, as seen in Fig. 9(b). Therefore, we observe spikes in the potential $v(R)$ [see Fig. 9(d)], which are also illustrated in another article on the same system [33].

The reason why the nodes of $\chi_{1}^{\mathrm{BO}}$ and $\chi_{2}^{\mathrm{BO}}$ are so close to each other can be better understood in a different basis. The potential part of the Hamiltonian can be diagonalized as

$$
\left(\begin{array}{ll}
V_{1}(R) & \gamma(R) \\
\gamma(R) & V_{1}(R)
\end{array}\right) \sim\left(\begin{array}{cc}
V_{-}(R) & 0 \\
0 & V_{+}(R)
\end{array}\right) .
$$

The wave function in the new adiabatic basis is represented by functions $\left(\chi_{-}(R), \chi_{+}(R)\right)$. The kinetic term in the new basis is no longer diagonal but is almost diagonal when the energetic separation of the potentials $V_{-}$and $V_{+}$is large. As the eigenenergy $E$ is always below $V_{+}$, we expect a low population of the state $\chi_{+}$. The coupled eigenvalue solution is then very close to the eigenvalue solution in just the potential $V_{-}(R)$. This can be seen in Fig. 9(c), where the seventh excited state of $V_{-}$is depicted. If $\chi_{+}(R)=0$, the marginal wave function $\chi(R)$ and the eigenfunction $\chi_{-}(R)$ would be identical and have nodes. However, for the exact calculation the kinetic term is not completely diagonal and therefore there is a nonzero contribution of $\chi_{+}(R)$, leading to a nonzero marginal wave function $\chi(R)=\sqrt{\chi_{-}(R)^{2}+\chi_{+}(R)^{2}}$.

\section{APPENDIX C: PROOF OF THE MAXNODE CONDITION}

The maxnode condition for one-dimensional (nondegenerate) problems is the following condition: The nodes of the $j$ th eigenstate $\chi_{0, j}$ of $v_{0}$ are at the positions where the energy $E_{i, j-i}\left(\boldsymbol{x}_{j}\right)$ of the $(j-i)$ th eigenstate $\chi_{i, j-i}$ of $v_{i}(i \leqslant j)$ has its maxima as a function of the spike positions $\boldsymbol{x}_{j}$. Here $v_{j}$ has $j$ spikes and $\boldsymbol{x}_{j}$ is the vector of the locations $x_{j, k}$ of those spikes with $k=1, \ldots, j$.

We prove the maxnode condition for a discretized problem only. Given is a Hermitian matrix $H$ of size $n \times n$ with spectrum $\lambda_{1}(H) \leqslant \lambda_{2}(H) \leqslant \cdots \leqslant \lambda_{n}(H)$. We construct the compression $H^{\prime}$ of size $m \times m$ from $H$ by removing some rows and columns. The Cauchy interlacing theorem provides the bounds $\lambda_{k}(H) \leqslant \lambda_{k}\left(H^{\prime}\right) \leqslant \lambda_{k+n-m}(H)$ for the eigenvalues of $H^{\prime}$.

We first consider the potential $v_{0}$ and the potential $v_{1}$ that only differs from $v_{0}$ by a spike at $x_{1,1}$. The Hamiltonian $H\left(v_{1}\right)$ for $v_{1}$ has one eigenvalue $+\infty$ and the corresponding eigenvector $\delta\left(x-x_{1,1}\right)$. Due to the orthogonality, all other eigenvectors of $H\left(v_{1}\right)$ are zero at $x_{1,1}$. We can thus obtain those other eigenvectors by solving the eigenvalue problem for $H^{\prime}\left(v_{1}\right)$ obtained from $H\left(v_{1}\right)$ by removing the row and column corresponding to $x_{1,1}$. As $H^{\prime}\left(v_{1}\right)$ is a compression of $H\left(v_{0}\right)$, the Cauchy interlacing theorem tell us that $\lambda_{k}\left(H\left(v_{0}\right)\right) \leqslant \lambda_{k}\left(H^{\prime}\left(v_{1}\right)\right) \leqslant \lambda_{k+1}\left(H\left(v_{0}\right)\right)$, i.e., the $k$ th eigenvalue of the spiky potential appears somewhere between the $k$ th and $(k+1)$ th eigenfunctions of the spikeless potential.

We also know that when $x_{1,1}$ is exactly at a node of the $(k+1)$ th eigenfunction of $v_{0}$, this function is also the $k$ th eigenfunction of $v_{1}$. Therefore, when $\lambda_{k}\left(H^{\prime}\left(v_{1}\right)\right)$ reaches its maximum as a function of $x_{1,1}$, this maximum is $\lambda_{k+1}\left(H\left(v_{0}\right)\right)$.

Next we consider $v_{2}$ that has two spikes at $\boldsymbol{x}_{2}=\left(x_{2,1}, x_{2,2}\right)$. Designating $H^{\prime}\left(v_{2}\right)$ as being the compressed Hamiltonian of $H\left(v_{2}\right)$ where the rows and columns associated with the two spikes have been removed, we have that $\lambda_{k}\left(H\left(v_{0}\right)\right) \leqslant$ 
$\lambda_{k}\left(H^{\prime}\left(v_{2}\right)\right) \leqslant \lambda_{k+2}\left(H\left(v_{0}\right)\right)$. Again, when $x_{2,1}$ and $x_{2,2}$ are exactly at nodes of the $(k+2)$ th eigenfunction of $v_{0}$, this function is also the $k$ th eigenfunction of $v_{2}$. Therefore, when $\lambda_{k}\left(H^{\prime}\left(v_{2}\right)\right)$ reaches its maximum as a function of $\boldsymbol{x}_{2}$, this maximum is $\lambda_{k+2}\left(H\left(v_{0}\right)\right)$. The maxnode condition follows by induction.

\section{APPENDIX D: ADIABATIC AND DIABATIC STATES}

We consider a transformation in the spirit of the transformation from adiabatic states to diabatic states in the Born-Oppenheimer framework, but for a potential that depends parametrically on the position of one spike. Figure 10 shows the seven lowest eigenstates $\chi_{1, k}\left(x_{1} ; x_{1,1}\right)$ and energies $E_{1, k}\left(x_{1,1}\right)$ of $v_{1}$ given in (58) with one spike at $x_{1,1}$, for the spikeless potential $v_{1}$ of (56). The left panels show the adiabatic ordering where the states are ordered according to their energetic order. The sign of $\chi_{1, k}\left(x_{1} ; x_{1,1}\right)$ along $x_{1,1}$ is undetermined and was set to be as continuous as possible, based on the overlap of $\chi_{1, k}$ on two neighboring grid points along $x_{1,1}$.

The adiabatic surfaces $E_{1, k}\left(x_{1,1}\right)$ do not cross, but a transformation similar to the transformation from adiabatic states to diabatic states in the Born-Oppenheimer approach is possible. Then the order is not based on the energy but such that the character of the wave function is continuously changing. The right panels of Fig. 10 show the result of a transformation to diabatic states. The transformation was achieved based on the continuity of $E_{1, k}\left(x_{1,1}\right)$ : Two neighboring points of $E_{1, k}\left(x_{1,1}\right)$ along the $x_{1,1}$ grid were used to linearly extrapolate the energy at the next point along $x_{1,1}$, and the true values of $E_{1, k}\left(x_{1,1}\right)$ were sorted according to this extrapolation. There are $2 \times 7$ diabatic states shown in the figure, seven that increase in energy from small to large $x_{1,1}$ and seven that decrease in energy.
[1] M. Born and R. Oppenheimer, Zur Quantentheorie der Molekeln, Ann. Phys. (Leipzig) 389, 457 (1927).

[2] G. Hunter, Conditional probability amplitudes in wave mechanics, Int. J. Quantum Chem. 9, 237 (1975).

[3] G. Hunter, Nodeless wave function quantum theory, Int. J. Quantum Chem. 17, 133 (1980).

[4] G. Hunter, Nodeless wave functions and spiky potentials, Int. J. Quantum Chem. 19, 755 (1981).

[5] A. Abedi, N. T. Maitra, and E. K. U. Gross, Exact Factorization of the Time-Dependent Electron-Nuclear Wave Function, Phys. Rev. Lett. 105, 123002 (2010).

[6] A. Abedi, N. T. Maitra, and E. K. U. Gross, Correlated electron-nuclear dynamics: Exact factorization of the molecular wavefunction, J. Chem. Phys. 137, 22A530 (2012).

[7] N. I. Gidopoulos and E. K. U. Gross, Electronic non-adiabatic states: Towards a density functional theory beyond the BornOppenheimer approximation, Philos. Trans. R. Soc. A 372, 20130059 (2014).

[8] A. Scherrer, F. Agostini, D. Sebastiani, E. K. U. Gross, and R. Vuilleumier, Nuclear velocity perturbation theory for vibrational circular dichroism: An approach based on the exact factorization of the electron-nuclear wave function, J. Chem. Phys. 143, 074106 (2015).

[9] A. Schild, F. Agostini, and E. K. U. Gross, Electronic flux density beyond the Born-Oppenheimer approximation, J. Phys. Chem. A 120, 3316 (2016).

[10] S. K. Min, F. Agostini, I. Tavernelli, and E. K. U. Gross, Ab initio nonadiabatic dynamics with coupled trajectories: A rigorous approach to quantum (de)coherence, J. Phys. Chem. Lett. 8, 3048 (2017).

[11] T. Fiedlschuster, J. Handt, E. K. U. Gross, and R. Schmidt, Surface hopping in laser-driven molecular dynamics, Phys. Rev. A 95, 063424 (2017).

[12] F. Talotta, S. Morisset, N. Rougeau, D. Lauvergnat, and F. Agostini, Internal conversion and intersystem crossing with the exact factorization, J. Chem. Theory Comput. 16, 4833 (2020).

[13] Y. Suzuki and K. Watanabe, Bohmian mechanics in the exact factorization of electron-nuclear wave functions, Phys. Rev. A 94, 032517 (2016).
[14] A. Scherrer, F. Agostini, D. Sebastiani, E. K. U. Gross, and R. Vuilleumier, On the Mass of Atoms in Molecules: Beyond the Born-Oppenheimer Approximation, Phys. Rev. X 7, 031035 (2017).

[15] R. Requist and E. K. U. Gross, Exact Factorization-Based Density Functional Theory of Electrons and Nuclei, Phys. Rev. Lett. 117, 193001 (2016).

[16] R. Requist, C. R. Proetto, and E. K. U. Gross, Exact factorization-based density functional theory of electronphonon systems, Phys. Rev. B 99, 165136 (2019).

[17] E. Khosravi, A. Abedi, and N. T. Maitra, Exact Potential Driving the Electron Dynamics in Enhanced Ionization of $\mathrm{H}_{2}^{+}$, Phys. Rev. Lett. 115, 263002 (2015).

[18] A. Abedi, E. Khosravi, and I. V. Tokatly, Shedding light on correlated electron-photon states using the exact factorization, Eur. Phys. J. B 91, 194 (2018).

[19] N. M. Hoffmann, H. Appel, A. Rubio, and N. T. Maitra, Lightmatter interactions via the exact factorization approach, Eur. Phys. J. B 91, 180 (2018).

[20] L. Lacombe and N. T. Maitra, Embedding via the Exact Factorization Approach, Phys. Rev. Lett. 124, 206401 (2020).

[21] A. Schild and E. K. U. Gross, Exact Single-Electron Approach to the Dynamics of Molecules in Strong Laser Fields, Phys. Rev. Lett. 118, 163202 (2017).

[22] J. Kocák and A. Schild, Many-electron effects of strong-field ionization described in an exact one-electron theory, Phys. Rev. Res. 2, 043365 (2020).

[23] J. Kocák, E. Kraisler, and A. Schild, Charge-transfer steps in density functional theory from the perspective of the exact electron factorization, J. Phys. Chem. Lett. 12, 3204 (2021).

[24] J. S. Briggs and J. M. Rost, Time dependence in quantum mechanics, Eur. Phys. J. D 10, 311 (2000).

[25] J. C. Arce, Unification of the conditional probability and semiclassical interpretations for the problem of time in quantum theory, Phys. Rev. A 85, 042108 (2012).

[26] J. S. Briggs, Equivalent emergence of time dependence in classical and quantum mechanics, Phys. Rev. A 91, 052119 (2015). 
[27] A. Schild, Time in quantum mechanics: A fresh look at the continuity equation, Phys. Rev. A 98, 052113 (2018).

[28] X. Gonze, J. S. Zhou, and L. Reining, Variations on the "exact factorization" theme, Eur. Phys. J. B 91, 224 (2018).

[29] H. P. Breuer and F. Petruccione, The Theory of Open Quantum Systems (Oxford University Press, Oxford, 2010).

[30] R. van Geleuken and A. V. Martin, Conditional wave theory of environmental interactions with a quantum particle, Phys. Rev. Res. 2, 033189 (2020).

[31] J. Czub and L. Wolniewicz, On the non-adiabatic potentials in diatomic molecules, Mol. Phys. 36, 1301 (1978).

[32] R. Lefebvre, Perturbations in vibrational diatomic spectra: Factorization of the molecular wave function, J. Chem. Phys. 142, 074106 (2015).

[33] R. Lefebvre, Factorized molecular wave functions: Analysis of the nuclear factor, J. Chem. Phys. 142, 214105 (2015).

[34] R. Lefebvre, Factorisation of zero-width resonance wave functions, Mol. Phys. 115, 1966 (2017).

[35] W. K. Peters, V. Tiwari, and D. M. Jonas, Nodeless vibrational amplitudes and quantum nonadiabatic dynamics in the nested funnel for a pseudo Jahn-Teller molecule or homodimer, J. Chem. Phys. 147, 194306 (2017).

[36] P. W. Foster and D. M. Jonas, Nonadiabatic eigenfunctions can have amplitude, signed conical nodes, or signed higher order nodes at a conical intersection with circular symmetry, J. Phys. Chem. A 121, 7401 (2017).

[37] P. W. Foster, W. K. Peters, and D. M. Jonas, Nonadiabatic eigenfunctions can have conical nodes, Chem. Phys. Lett. 683, 268 (2017).
[38] W. M. C. Foulkes, R. Q. Hood, and R. J. Needs, Symmetry constraints and variational principles in diffusion quantum Monte Carlo calculations of excited-state energies, Phys. Rev. B 60, 4558 (1999).

[39] A. Schild, QMstunfti: Quantum dynamics with (sparse) matrix representations in Python, https://gitlab.com/axelschild/ QMstunfti.

[40] E. Jones, T. Oliphant, P. Peterson and others, SciPy: Open Source Scientific Tools for Python, http://www.scipy.org.

[41] R. B. Lehoucq, D. C. Sorensen, and C. Yang, ARPACK Users' Guide: Solution of Large-Scale Eigenvalue Problems with Implicitly Restarted Arnoldi Methods (SIAM, Philadelphia, 1998).

[42] R. Requist, F. Tandetzky, and E. K. U. Gross, Molecular geometric phase from the exact electron-nuclear factorization, Phys. Rev. A 93, 042108 (2016).

[43] B. F. E. Curchod and F. Agostini, On the dynamics through a conical intersection, J. Phys. Chem. Lett. 8, 831 (2017).

[44] X. L. Yang, S. H. Guo, F. T. Chan, K. W. Wong, and W. Y. Ching, Analytic solution of a two-dimensional hydrogen atom. I. Nonrelativistic theory, Phys. Rev. A 43, 1186 (1991).

[45] F. Zahariev, M. S. Gordon, and M. Levy, Nodal variational principle for excited states, Phys. Rev. A 98, 012144 (2018).

[46] K. Dressler, The lowest valence and Rydberg states in the dipole-allowed absorption spectrum of nitrogen. A survey of their interactions, Can. J. Phys. 47, 547 (1969). 